\title{
Strong Recurrent Networks Compute the Orientation Tuning of Surround Modulation in the Primate Primary Visual Cortex
}

\author{
S. Shushruth, ${ }^{1}$ Pradeep Mangapathy, ${ }^{1}$ Jennifer M. Ichida, ${ }^{1}$ Paul C. Bressloff, ${ }^{2}$ Lars Schwabe, ${ }^{3 *}$ \\ and Alessandra Angelucci ${ }^{1 *}$ \\ 1Department of Ophthalmology and Visual Science, Moran Eye Center and 2Department of Mathematics, University of Utah, Salt Lake City, Utah 84132, and \\ ${ }^{3}$ Department of Computer Science and Electrical Engineering, University of Rostock, 18059 Rostock, Germany
}

In macaque primary visual cortex (V1), neuronal responses to stimuli inside the receptive field (RF) are modulated by stimuli in the RF surround. This modulation is orientation specific. Previous studies suggested that, for some cells, this specificity may not be fixed but changes with the stimulus orientation presented to the RF. We demonstrate, in recording studies, that this tuning behavior is instead highly prevalent in V1 and, in theoretical work, that it arises only if V1 operates in a regime of strong local recurrence. Strongest surround suppression occurs when the stimuli in the RF and the surround are iso-oriented, and strongest facilitation when the stimuli are cross-oriented. This is the case even when the RF is suboptimally activated by a stimulus of nonpreferred orientation but only if this stimulus can activate the cell when presented alone. This tuning behavior emerges from the interaction of lateral inhibition (via the surround pathways), which is tuned to the preferred orientation of the RF, with weakly tuned, but strong, local recurrent connections, causing maximal withdrawal of recurrent excitation at the feedforward input orientation. Thus, horizontal and feedback modulation of strong recurrent circuits allows the tuning of contextual effects to change with changing feedforward inputs.

\section{Introduction}

Surround modulation in primary visual cortex (V1) is orientation specific (Blakemore and Tobin, 1972; Nelson and Frost, 1978; Allman et al., 1985; Gilbert and Wiesel, 1990). This specificity is thought to underlie perceptual "figure-ground" segmentation (Malik and Perona, 1990; Knierim and van Essen, 1992; Lamme, 1995; Li, 1999), or detection of salient targets (Petrov and McKee, 2006). Most previous studies examined the orientation specificity of surround modulation by stimulating the receptive field (RF) with gratings at the preferred orientation of the cell, while varying the orientation of gratings in the surround. They found maximal suppression when the surround stimulus was at the preferred orientation of the cell and less suppression or facilitation when it was at the orthogonal-to-preferred orientation (Blakemore and Tobin, 1972; DeAngelis et al., 1994; Levitt and Lund, 1997; Sengpiel et al., 1998; Cavanaugh et al., 2002b; Müller et al., 2003). This observation, and the lack of tuned suppression in the LGN (Solomon et al., 2002; Webb et al., 2002), led

\footnotetext{
Received July 21, 2011; revised Nov. 3, 2011; accepted Nov. 6, 2011.

Author contributions: S.S., P.C.B., L.S., and A.A. designed research; S.S., P.M., J.M.I., L.S., and A.A. performed research; S.S. analyzed data; S.S., L.S., and A.A. wrote the paper.

We are grateful for support from National Science Foundation Grants IBN-0344569 and IOS-0848106 (A.A.), National Institute of Health Grants EY015262 (A.A.) and EY015609 (J.M.I.), and by a grant from Research to Prevent Blindness (to the Department of Ophthalmology, University of Utah, Salt Lake City, UT).

*L.S. and A.A. contributed equally to this work.

Correspondence should be addressed to Alessandra Angelucci, 65 Mario Capecchi Drive, Salt Lake City, UT 84132. E-mail: alessandra.angelucci@hsc.utah.edu.

DOI:10.1523/JNEUROSCI.3789-11.2012

Copyright $\odot 2012$ the authors $\quad 0270-6474 / 12 / 320308-14 \$ 15.00 / 0$
}

to the suggestion (Angelucci and Bressloff, 2006) that intracortical horizontal connections (HCs) and feedback (FB) connections, linking neurons with similar orientation preference (Malach et al., 1993; Shmuel et al., 2005; but see Stettler et al., 2002), generate the orientation specificity of surround modulation in $\mathrm{V} 1$.

Two previous studies (Sillito et al., 1995; Cavanaugh et al., $2002 \mathrm{~b}$ ), instead, using stimuli of changing orientation in both the $\mathrm{RF}$ and surround, reported for few V1 cells that the orientation specificity of surround suppression is independent of the preferred orientation of the cell but changes depending on the stimulus orientation inside the RF. However, it remains unclear whether only a subclass of V1 cells shows this effect. Understanding the prevalence of this tuning behavior in V1 is important because it could serve in computation of visual saliency. Furthermore, this tuning cannot be generated solely by intracortical connections with fixed orientation specificities. Therefore, understanding its mechanism is crucial to understand cortical computations. Finally, the orientation tuning of surround facilitation remains controversial. Sillito et al. (1995) reported that any orientation discontinuity in the RF and surround evoked facilitation, whereas Cavanaugh et al. (2002b) found little evidence for facilitation.

We have examined the orientation tuning of surround suppression and facilitation in a large population of V1 neurons by recording responses to gratings of varying orientation in the RF and surround. We find that, for most V1 cells, the tuning of surround modulation depends on the stimulus orientation presented to the RF, provided this stimulus can activate the cell when presented alone. Suppression is maximal for iso-oriented stimuli 
in the RF and surround, facilitation for cross-oriented stimuli. Facilitation occurs less frequently than suppression but emerges in many cells when the RF and surround are suboptimally stimulated.

Using a simple recurrent network model, we show that this tuning behavior can be achieved only if $\mathrm{V} 1$ operates in a regime of strong but balanced recurrent excitation and inhibition, and it results from the interaction of two differently tuned intracortical mechanisms. Tuned lateral inhibition (via the surround pathways) of untuned local recurrent connections causes maximal withdrawal of recurrent excitation at the feedforward-input orientation, resulting in the stimulus-dependent tuning of the surround.

\section{Materials and Methods}

Surgical preparation and recording. We recorded extracellularly from parafoveal V1 of five anesthetized (sufentanil citrate, 4-12 $\mu \mathrm{g}$. $\mathrm{kg}^{-1} \cdot \mathrm{h}^{-1}$ ) and paralyzed (vecuronium bromide, $0.1-0.3 \mu \mathrm{g} \cdot \mathrm{kg}^{-1}$. $\mathrm{h}^{-1}$ ) macaque monkeys (Macaca fascicularis; three females and two males). All procedures conformed to the guidelines of the University of Utah Institutional Animal Care and Use Committee. Animals were artificially respirated with a 30:70 mixture of $\mathrm{O}_{2}$ and $\mathrm{N}_{2} \mathrm{O}$. Electrocardiogram was continuously monitored, and end tidal $\mathrm{CO}_{2}$ was maintained at $30-33 \mathrm{mmHg}$, rectal temperature near $37^{\circ} \mathrm{C}$, and blood oxygenation near $100 \%$. The pupils were dilated with topical atropine, the corneas were protected with rigid gas-permeable contact lenses, and the eyes were refracted. The locations of the foveae were plotted at the beginning of the experiment and periodically thereafter, using a reversible ophthalmoscope.

Single-unit recordings were made with Epoxylite-coated tungsten microelectrodes (4-6 M 2 ; FHC). Spikes were conventionally amplified, bandpass filtered between $400 \mathrm{~Hz}$ and $5 \mathrm{kHz}$, and sampled at $22 \mathrm{kHz}$ by a dual-processor G5 Power Macintosh computer running custom software (EXPO) courtesy of Dr. Peter Lennie (University of Rochester, Rochester NY). Spikes were displayed on a monitor, and templates for discriminating spikes were constructed by averaging multiple traces. The timing of waveforms that matched the templates was recorded with an accuracy of $0.1 \mathrm{~ms}$.

Visual stimuli. Drifting sinusoidal gratings of the same mean luminance as the background and of $80 \%$ contrast were generated by the same software and computer that recorded spikes and were displayed on a calibrated monitor (Sony GDM-C520K) refreshed at $100 \mathrm{~Hz}$, of mean luminance $\sim 45.7 \mathrm{~cd} / \mathrm{m}^{2}$, at a viewing distance of $57 \mathrm{~cm}$. For each cell, we first determined the preferred orientation, drift direction, and spatial and temporal frequencies. Then the area and center of the minimum response field (mRF) were carefully located quantitatively using a grating patch of $0.2^{\circ}$ diameter. The area of the mRF was defined as the visual field region in which the small grating patch elicited a response at least 2 SDs above the spontaneous rate, and the geometric center of this area was defined as the mRF center. We performed spatial summation measurements at $80 \%$ contrast levels, using circular patches of drifting gratings of increasing diameter centered over the mRF of the cell. The patch diameter ranged from 0.2 to $26^{\circ}$ and consisted of 16 diameters (in steps of $0.2^{\circ}$ from 0.2 to $1.2^{\circ}$ and then $1.5,1.8,2,2.4,3.6$, $\left.5,10,15,20,26^{\circ}\right)$. From these size-tuning curves for each cell, we extracted as a measure of RF size the patch diameter at peak response. Cells that showed no surround effects (i.e., that asymptoted at the peak response) were discarded.

Characterization of the surround orientation tuning. We recharacterized the orientation tuning of the RF of each neuron using the optimal stimulus size for the recorded neuron, derived from the spatial summation measurements described above. From this orientation-tuning curve, we determined four orientation values: optimal (Opt), orthogonal-to-optimal (Ortho), suboptimal (Sub), and suboptimal-weak $\left(\mathrm{Sub}_{\mathrm{WK}}\right)$. $\mathrm{Sub}_{\mathrm{WK}}$ was defined as the orientation evoking a response $<50 \%$ of maximum but $>2$ SDs above spontaneous activity, whereas Sub was the orientation evoking a response halfway between those evoked by Opt and $\mathrm{Sub}_{\mathrm{WK}}$ orientations (see Fig. $2 B, E$ ). Thus, the actual orientation in degrees for each of these four orientation values varied from cell to cell. Cells untuned for stimulus orientation, i.e., those for which all stimulus orientations evoked similar response rates, were not characterized further because there were no suboptimal orientations for such cells.

The stimulus (see Fig. $2 \mathrm{~A}$ ) used to characterize the orientation tuning of surround modulation consisted of a center grating matched to the RF diameter of the cell, surrounded by an annular grating with a fixed outer diameter $\left(26^{\circ}\right)$ and an inner diameter of decreasing size, from 24 to $5^{\circ}$ (for a total of nine inner diameter sizes: 5, 6, 8, 10, 12, 14, 16, 20, and $24^{\circ}$ ). The surround region $<5^{\circ}$ in diameter, which we term the "near" surround, was masked by a blank annulus of the same luminance as the background interposed between the center grating patch and the surround annular grating. The near surround was shown previously to be coextensive with the average spatial spread of monosynaptic HCs (Angelucci et al., 2002) and with the largest surround fields of LGN afferents (Sceniak et al., 2006; Alitto and Usrey, 2008). We used this stimulus to isolate the modulatory signals from the "far" surround (see Fig. 2A) (Ichida et al., 2007; Schwabe et al., 2010), i.e., the surround region extending beyond the spread of monosynaptic HCs, presumed to be mediated by FB connections (Angelucci et al., 2002). Center and surround orientations were presented at all combinations of the four aforementioned orientations (Opt, Sub, $\mathrm{Sub}_{\mathrm{WK}}$, and Ortho), in pseudorandom order. Each stimulus consisted of an initial presentation of the center grating lasting $200 \mathrm{~ms}$, followed by the appearance of the surround grating, and the two persisted for an additional $300 \mathrm{~ms}$. The delayed presentation enabled us to study the modulation on the steady-state response of the neuron and to avoid ambiguities caused by the nonspecific response onset of the neuron. The short presentation time is compatible with a physiologically relevant time window because the mean duration of saccades is $\sim 350 \mathrm{~ms}$ (Gallant et al., 1998). Each stimulus was repeated for $8-16$ trials. Control conditions included a blank screen, of the same luminance as the background, for a measure of spontaneous activity, center-alone conditions at all orientations for a baseline response, and surround annulus-alone conditions at all sizes at Opt orientation to ensure that the surround stimulus alone did not drive a response from the cell.

Data analysis. To characterize orientation-tuning curves for the RFs of our cell population, we used as a measure of orientation selectivity, the circular variance (CV) (Cavanaugh et al., 2002b; Ringach et al., 2002). The circular variance for each cell was calculated as follows:

$$
\mathrm{CV}=\frac{\left|\sum_{n}\left(R_{n}\right) \exp \left(i 2 \theta_{n}\right)\right|}{\sum_{n}\left(R_{n}\right)}
$$

where $R_{n}$ is the response magnitude of the neuron to stimulus orientation $\theta_{n}$. For cells with orientation and direction selectivity, we calculated CV by replacing $2 \theta_{n}$ with $\theta_{n}$ and took the maximum of the two values. The $\mathrm{CV}$ has a value of 0 for a dataset falling uniformly on a circle (not orientation selective) and a value of 1 for a dataset with response only to a single orientation/direction (highly selective).

All analyses of surround modulation, unless mentioned otherwise, were performed for the $300 \mathrm{~ms}$ time window after the appearance of the surround stimulus. Spike trains were imported into MATLAB and analyzed using custom scripts. Statistical tests used to determine significance are reported in Results.

The model. The model is a mean-field recurrent network model of recurrently connected orientation columns (an orientation "hypercolumn"). The feedforward input and the inputs from the surround via HCs and FB connections are described as additional input currents, i.e., here we do not model recurrent connections between center and surround but focus on the effects of the external input from the surround on the activity within the hypercolumn.

Let $r_{\alpha}\left(\theta_{i}, t\right)$ denote the activity of neurons of type $\alpha \in\{$ EXC,BSK $\}$, corresponding to excitatory pyramidal and inhibitory basket neurons, respectively, with a "preferred orientation" $\theta_{i}$ (a label attached to each column to establish feedforward and recurrent connectivity), 
Table 1. Parameter values

\begin{tabular}{|c|c|c|c|}
\hline Parameter & Description & Value in the recurrent model & Value in the feedforward model \\
\hline$\tau_{\mathrm{EXC}}$ & Time constant & $10 \mathrm{~ms}$ & Same \\
\hline$\tau_{\mathrm{BSK}}$ & Time constant & $6 \mathrm{~ms}$ & Same \\
\hline $\mathrm{T}_{\mathrm{EXC}}=\mathrm{T}_{\mathrm{BSK}}$ & Threshold & 0.5 & Same \\
\hline $\mathrm{g}_{\mathrm{EXC}}=\mathrm{g}_{\mathrm{BSK}}$ & Single-neuron gain & 1 & Same \\
\hline$W_{\mathrm{EXC}}^{\mathrm{FF}}$ & Feedforward excitation & 1 & Same \\
\hline$W_{\mathrm{BSK}}^{\mathrm{FF}}$ & Feedforward excitation & 1 & Same \\
\hline$W_{\text {EXC,EXC }}^{\mathrm{LOC}}$ & Local excitation of excitatory neurons & 20 & 0 \\
\hline$W_{\mathrm{BSK}, \mathrm{EXC}}^{\mathrm{LOC}}$ & Local excitation of basket neurons & 20 & 0 \\
\hline$W_{\mathrm{EXC}, \mathrm{BSK}}^{\mathrm{LOC}}$ & Inhibition of excitation via basket neurons & -24 & 0 \\
\hline$W_{B S K, B S K}^{\mathrm{LOC}}$ & Inhibition of basket neurons via basket neurons & -24 & 0 \\
\hline$W_{\mathrm{EXC}}^{\mathrm{MOD}}$ & Surround modulation of excitatory neurons & -0.01 & -0.2 \\
\hline$W_{\mathrm{BSK}}^{\mathrm{MOD}}$ & Surround modulation of basket neurons & 0.02 & 0 \\
\hline$\kappa_{\mathrm{EXC}}^{\mathrm{FF}}=\kappa_{\mathrm{BSK}}^{\mathrm{FF}}$ & Tuning of feedforward input & 0.5 & Same \\
\hline$\kappa_{\mathrm{EXC}, \mathrm{EXC}}^{\mathrm{LOC}}=\kappa_{\mathrm{BSK}, \mathrm{EXC}}^{\mathrm{LOC}}$ & Tuning of recurrent excitatory projections & 0.2 & Not relevant \\
\hline$\kappa_{\mathrm{EXC}, \mathrm{BSK}}^{\mathrm{LOC}}=\kappa_{\mathrm{BSK}, \mathrm{BSK}}^{\mathrm{LOC}}$ & Tuning of recurrent basket neuron projections & 0.2 & Not relevant \\
\hline$\kappa_{\mathrm{EXC}}^{\mathrm{MOD}}=\kappa_{\mathrm{BSK}}^{\mathrm{MOD}}$ & Tuning of surround modulation & 0.5 & 0.5 \\
\hline
\end{tabular}

$1 \leq i \leq N$ and $\theta_{i}=(i-1) 180 / N$, where $N=32$ is the number of orientation columns. Then, the dynamics of the activities are given by the following:

$$
\begin{aligned}
& \tau_{\alpha} \frac{d}{d t} r_{\alpha}\left(\theta_{i}, t\right)=-r_{\alpha}\left(\theta_{i}, t\right)+g_{\alpha}\left[I_{\alpha}^{\mathrm{FF}}\left(\theta_{i}, \phi^{\mathrm{ctr}}\right)+I_{\alpha}^{\mathrm{LOC}}\left(\theta_{i}\right)\right. \\
& \left.+I_{\alpha}^{\mathrm{MOD}}\left(\theta_{i}, \phi^{\mathrm{sur}}\right)-T_{\alpha}\right]^{+} \\
& I_{\alpha}^{\mathrm{FF}}\left(\theta_{i}, \phi^{\mathrm{ctr}}\right)=W_{\alpha}^{\mathrm{FF}} \frac{\exp \left(\kappa_{\alpha}^{\mathrm{FF}} \cdot \cos \left(2 d\left(\theta_{i}, \phi^{\mathrm{ctr}}\right)\right)\right)}{\max \exp \left(\kappa_{\alpha}^{\mathrm{FF}} \cdot \cos \left(2 d\left(\theta_{j}, \phi^{\mathrm{ctr}}\right)\right)\right)} \\
& I_{\alpha}^{\mathrm{LOC}}\left(\theta_{i}\right)=\sum_{\beta} \sum_{j} W_{\alpha \beta}^{\mathrm{LOC}} \frac{\exp \left(\kappa_{\alpha \beta}^{\mathrm{LOC}} \cdot \cos \left(2 d\left(\theta_{i}, \theta_{j}\right)\right)\right)}{\sum_{j} \exp \left(\kappa_{\alpha \beta}^{\mathrm{LOC}} \cdot \cos \left(2 d\left(\theta_{i}, \theta_{j}\right)\right)\right)} \\
& I_{\alpha}^{\mathrm{MOD}}\left(\theta_{i}, \phi^{\text {sur }}\right)=W_{\alpha}^{\mathrm{MOD}} \frac{\exp \left(\kappa_{\alpha}^{\mathrm{MOD}} \cdot \cos \left(2 d\left(\theta_{i}, \phi^{\text {sur }}\right)\right)\right)}{\max \exp \left(\kappa_{\alpha}^{\mathrm{MOD}} \cdot \cos \left(2 d\left(\theta_{j}, \phi^{\text {sur }}\right)\right)\right)} \\
& d\left(\theta_{1}, \theta_{2}\right)=\min \left(180-\left|\theta_{1}-\theta_{2}\right|,\left|\theta_{1}-\theta_{2}\right|\right)
\end{aligned}
$$

where $\tau_{\alpha}$ is a membrane time constant, $g_{\alpha}$ is the gain of the thresholdlinear firing rate function, $T_{\alpha}$ is a threshold, and the function $[u]^{+}$is defined as $u$ for $u>0$ and 0 otherwise. The tuning of the feedforward input, $I_{\alpha}^{\mathrm{FF}}$, the local recurrent inputs, $I_{\alpha}^{\mathrm{LOC}}$, and the modulatory inputs, $I_{\alpha}^{\mathrm{MOD}}$, are given by circular functions with parameters $\kappa$, where high values correspond to sharply tuned inputs and lower values to weakly tuned inputs. Parameter values are given in Table 1. Note, however, that although we are modeling explicitly only excitatory pyramidal and inhibitory basket neurons, $\alpha, \beta \in\{\mathrm{EXC}, \mathrm{BSK}\}$, we assume a canonical microcircuit with a high-threshold inhibitory neuron (Bressloff and Cowan, 2002; Schwabe et al., 2006), which projects only locally within an orientation column to the excitatory neurons (see Fig. 8 A, Top). The effect of this neuron is included into a negative modulatory input, $W_{\mathrm{EXC}}^{\mathrm{MOD}}<0$, from the surround. Numerical integration is performed via a RungeKutta method (function ode45 in MATLAB; MathWorks). The full MATLAB source is available at http://senselab.med.yale.edu.

\section{Results}

\section{Orientation dependence of surround modulation}

We recorded extracellularly from a total of 126 single units ( 4 had simple and 122 had complex RFs) in parafoveal V1 (between 2 and $5.5^{\circ}$ eccentricity; median eccentricity, $3.75^{\circ}$ ) from five anesthetized macaque monkeys. Our stimuli were drifting sinusoidal gratings of $80 \%$ contrast whose spatial and temporal frequency

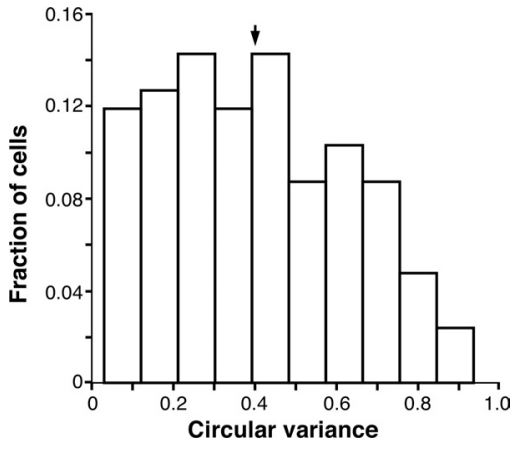

Figure 1. Orientation selectivity of the RF for the neuronal population. Distribution of circular variance values (a measure of orientation selectivity) for the RF responses of our neuronal sample. Arrow, Mean.

were optimized for the recorded cell. Our cell population encompassed a wide range of orientation selectivities, from broadly tuned to sharply tuned cells. Specifically, the circular variance of the RF response (a measure of orientation selectivity; see Materials and Methods) for the population ranged from 0.03 to 0.93 (mean, 0.4; Fig. 1). RF diameters ranged from 0.2 to $1.8^{\circ}(0.2-$ $0.8^{\circ}$ for $60.3 \%$ of cells; median, $\left.0.8^{\circ}\right)$. To probe surround modulation, we used the stimulus illustrated in Figure $2 \mathrm{~A}$. This consisted of a center grating patch optimized to the RF size of the cell and a surround annular grating of $26^{\circ}$ fixed outer diameter, and an inner diameter whose size was systematically decreased from 24 to $5^{\circ}$. The surround region $<5^{\circ}$ in diameter, i.e., the near surround, was masked by a blank annulus surrounding the center grating. We varied the size of the surround stimulus because we wanted to investigate the tuning of both facilitatory and suppressive effects from the surround, and we showed previously that the former are better revealed using small surround stimuli, whereas suppression dominates for large surround stimuli (Ichida et al., 2007).

To assay for the orientation tuning of surround modulation, we first characterized the orientation tuning curve of the RF response using a grating optimized to the RF size of the recorded cell. From this, we selected four orientations: Opt, Ortho, Sub, $S_{\mathrm{Sub}}$, with $\mathrm{Sub}_{\mathrm{WK}}$ evoking a weaker response from the cell than Sub (see Materials and Methods and Fig. 2B,E). For each sur- 


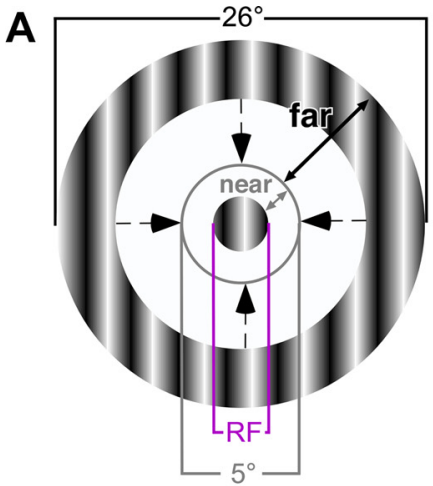

B
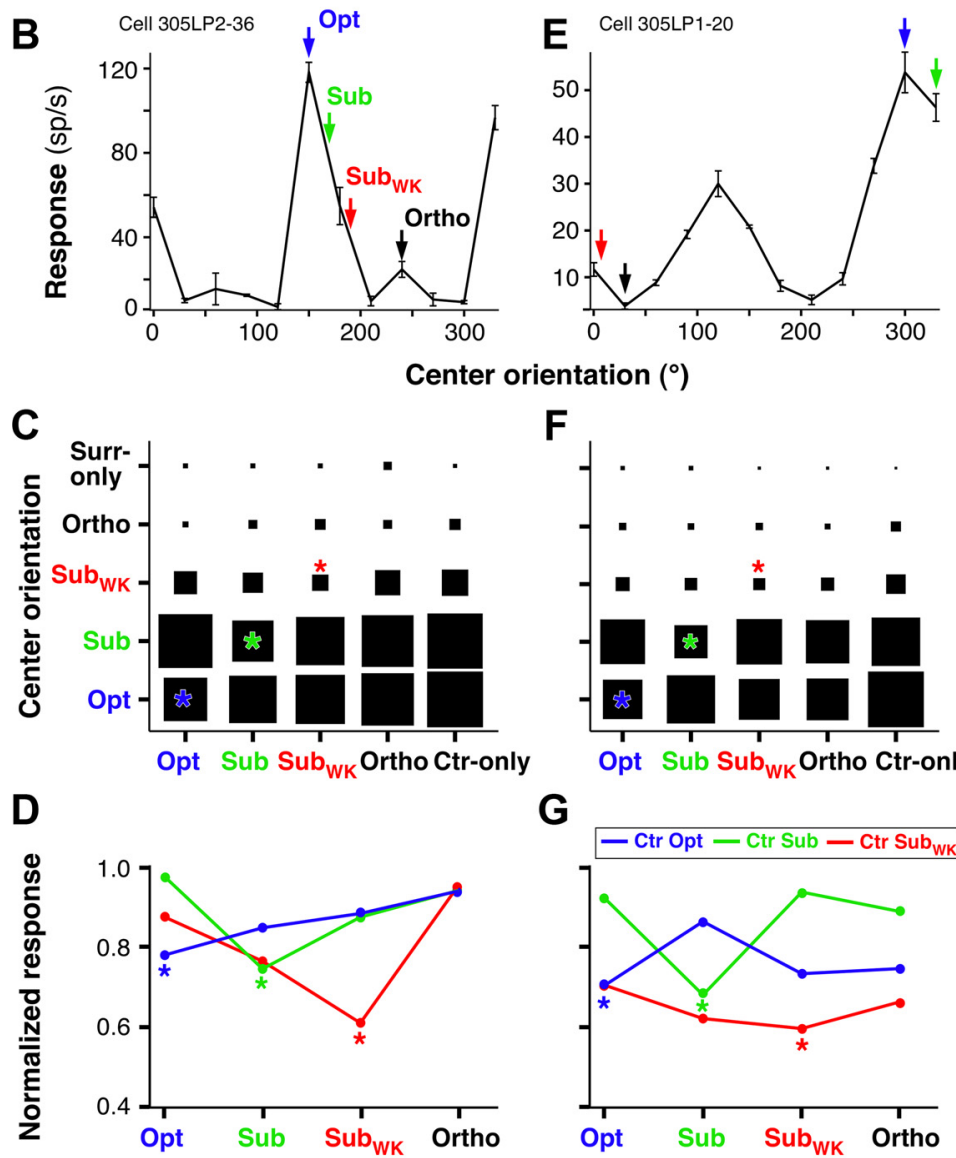

$\mathbf{F}$

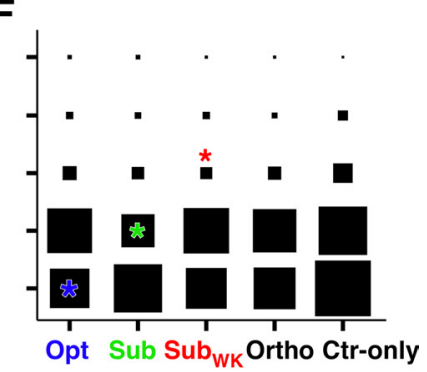

G

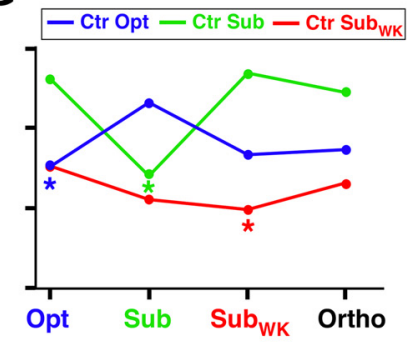

Surround orientation

Figure 2. Response of two example V1 cells to center and large surround gratings of changing orientation. $A$, Stimulus used to characterize the orientation specificity of surround modulation. The center grating was matched to the RF size, and the surround grating varied in size and was always separated from the center grating by a blank gap $\geq 5^{\circ}$ diameter. "near" and "far" indicate the size of the near and far surround, as defined in this study. $\boldsymbol{B}$, Orientation tuning curve of the RF response for an example V1 cell. The arrows indicate the four orientations chosen for this cell to characterize the orientation specificity of surround modulation. Error bars are SEM. $\boldsymbol{C}$, Response of the same example cell as in $\boldsymbol{B}$ to center and surround gratings presented at the four orientations indicated by arrows in $\boldsymbol{B}$. Ctr-only, Center-only response, i.e., in the absence of a surround stimulus; Surr-only, surround-only response, i.e., in the absence of a center stimulus. All responses are normalized to the center-only response at the 0 pt orientation for this cell. Asterisks indicate responses to center and surround stimuli of the same orientation. $\boldsymbol{D}$, For each of three different center orientations, indicated as curves of different colors, we show the response of the same cell as in $\boldsymbol{B}$ and $\boldsymbol{C}$ to center-surround stimuli as a function of surround stimulus orientation. Here responses are normalized to the center-only response at each respective orientation. Asterisks mark the iso-orientation conditions. $\boldsymbol{E}-\mathbf{G}$, Same as in $\boldsymbol{B}-\boldsymbol{D}$ but for a different example cell.

round annulus inner diameter, the center and surround gratings were presented at all combinations of these four orientations. Not all conditions could be assayed for each cell because occasionally a cell was lost before completing this long stimulation protocol.
In addition, we discarded all conditions in which the center-only response was $<2$ SDs above spontaneous activity. This reduced the sample size to 111 cells for the Opt center condition, 105 for Sub, 99 for Sub $_{\mathrm{WK}}$, and 13 for Ortho. Most cells did not respond above this baseline criterion in the Ortho center condition, with or without a surround stimulus (Fig. 2C,F); therefore, we did not include this condition in our comparative analysis.

\section{Orientation specificity of}

surround suppression

To measure the orientation specificity of surround suppression, we specifically analyzed neuronal responses to the largest surround stimuli used (i.e., the surround annuli with the smallest inner diameter). This is because large surround stimuli typically evoke suppression (DeAngelis et al., 1994; Sceniak et al., 2001; Cavanaugh et al., 2002b; Levitt and Lund, 2002; Ichida et al., 2007). Figure 2, $C$ and $F$, shows the response of two representative neurons to center and surround gratings presented at all combinations of four different orientations (Opt, Sub, $\mathrm{Sub}_{\mathrm{wK}}$, and Ortho). The area of each square in the matrix corresponds to the firing rate (spikes per second) of the cell in response to that particular combination of center and surround orientations, averaged across the three smallest surround annulus inner diameters, and normalized to the centeronly response at the Opt orientation. Center-only and surround-only responses at different orientations were also normalized to the Opt center-only response. For both cells, maximal suppression occurred when the surround stimulus was at the same orientation as the center stimulus (the iso-orientation conditions are marked by asterisks in Fig. $2 C, F$ ). This was regardless of the center stimulus orientation, as long as the latter could evoke a significant response from the cell when presented alone. Thus, a center stimulus presented at the Ortho orientation evoked no significant response from the cell with or without a surround stimulus, and this was consistent across the population. Figure 2, $D$ and $G$, plots the responses of the same two cells in a different format, i.e., as normalized response as a function of surround stimulus orientation. Colored curves correspond to different center orientation conditions, and the cell response (averaged across the three smallest surround inner diameters) was normalized to the center-only response at the corresponding orientation. The iso-orientation conditions (marked by an asterisk) were the most suppressive regardless of center stimulus orientation. 
Similar results were observed across the population. Figure $3 A-C$ shows for each center orientation (Opt, Sub, and $\mathrm{Sub}_{\mathrm{WK}}$ in $A, B$, and $C$, respectively) scatter plots of the response to center-surround stimuli of the same orientation (on the $x$-axes) versus the response to centersurround stimuli of different orientation (on the $y$-axes). Responses are averages of the three smallest surround annulus inner diameters used, normalized to the respective center-only response (i.e., to the Opt center-only response in $A$, to the Sub center-only response in $B$, etc.). In $A-C$, the majority of points lie above the diagonal ( $A, 72 \%$ of green points, $81 \%$ of red, and $78 \%$ of black; $B, 85 \%$ blue, $76 \%$ red, and $83 \%$ black; $C, 80 \%$ blue, $69 \%$ green, and $43 \%$ black), indicating that for most cells the iso-orientation condition was the most suppressive. To better illustrate the relation between the different surround orientations, Figure $3 D-F$ shows for each center orientation (Opt in $D$, Sub in $E$, and $\operatorname{Sub}_{\mathrm{WK}}$ in $F$ ) the distribution of responses to each of three different surround stimulus orientations (color coded). Response for each cell was expressed as the ratio of the response in the iso-orientation condition to the response in the non-isoorientation condition. In $D-F$, the ratio for most cells is $<1$, indicating that, regardless of center stimulus orientation, the centersurround iso-orientation condition evoked the smallest response from the cell. All mean ratios (Fig. 3D-F, arrows) were significantly different from 1 (see legend to Fig. 3D-F for mean and $p$ values), except for the Ortho surround condition in $F$, likely because of the small orientation difference between a surround stimulus of Ortho and one of $\mathrm{Sub}_{\mathrm{WK}}$ orientation.

Figure $3 D-F$ also demonstrates that surround stimulus orientations nearest the orientation of the center stimulus evoked stronger suppression than surround orientations farthest from the center orientation. Again, this was the case regardless of center stimulus orientation. Thus, for example, when the center stimulus was at Opt (Fig. 3D), Sub stimuli in the surround (green bars) evoked on average stronger suppression than Ortho surround stimuli (black bars). Specifically, the mean ratio (response at iso-orientation/ response at non-iso-orientation) for Sub surround stimuli was significantly larger than the mean ratio for Ortho surround stimuli ( $p=0.03$, Student's $t$ test). Instead, when the center stimulus was at $\operatorname{Sub}_{\mathrm{WK}}$ (Fig. $3 F$ ), an Ortho surround orientation (which is closest to $\mathrm{Sub}_{\mathrm{WK}}$ ) evoked significantly stronger suppression than an Opt surround (which is farthest in orientation from $\mathrm{Sub}_{\mathrm{WK}}$ ); the mean ratio for Ortho surround stimuli was significantly larger than the mean ratio for Opt surround stimuli $(p=0.0016)$.

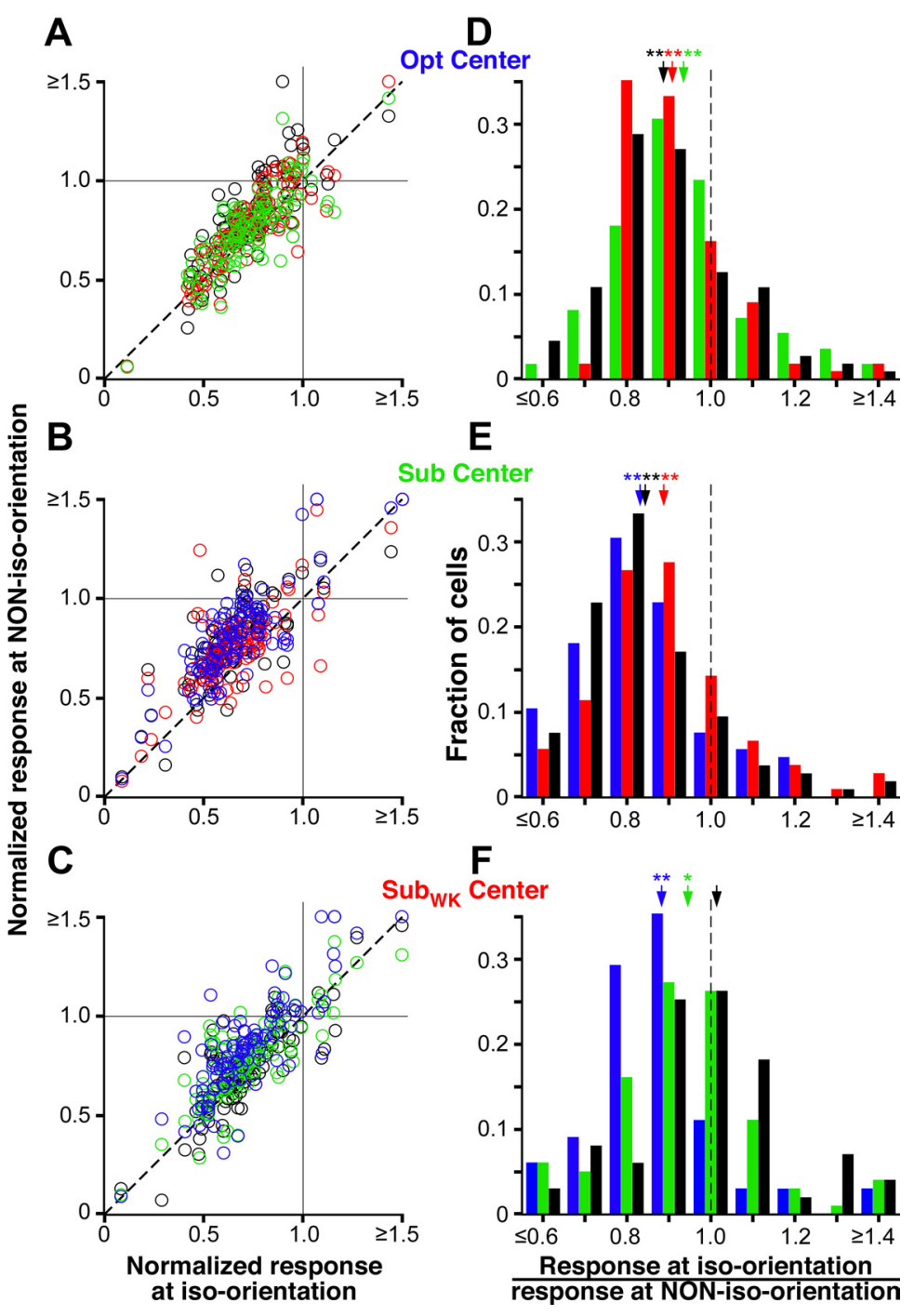

Figure 3. Orientation specificity of surround suppression: population data. $\boldsymbol{A}-\boldsymbol{C}$, For each cell, the normalized response to the iso-orientation condition (i.e., center and surround stimulus presented at the orientation indicated at the top right of each respective panel) is plotted against the normalized response to each non-iso-orientation condition (i.e., center stimulus at the orientation indicated at the top right of the panel and surround stimulus at each of 3 different orientations). The normalized response was estimated as the response to a particular combination of center and surround stimulus orientation, averaged across the three largest surround stimulus sizes used, and normalized to each respective center-only response. The different surround orientations are color coded as indicated in the legend at the top of the figure. Normalized responses $>1$ indicate that the surround stimulus facilitated the response of the cell to the center stimulus. Most cells, however, showed responses $<1$, i.e., were suppressed by surround stimuli of any orientation. $\boldsymbol{D}-\boldsymbol{F}$, For each center stimulus orientation (Opt in $\boldsymbol{D}$, Sub in $\boldsymbol{E}$, and $\operatorname{Sub}_{\mathrm{WK}}$ in $\boldsymbol{F}$ ), the distribution of responses to three different surround orientations are plotted (color coded as indicated in the legend at the top of the figure). Responses are expressed as response to iso-oriented center-surround stimuli divided by the response to non-isooriented center-surround stimuli. A ratio $<1$ indicates that the iso-orientation condition evoked a weaker response (i.e., stronger suppression) than the non-iso-orientation condition. Arrows, Means. Mean ratios \pm SEM are as follows: $\boldsymbol{D}, 0.94 \pm 0.02$ (green, Sub), $0.91 \pm 0.01$ (red, Sub wK $_{\text {) }}, 0.89 \pm 0.01$ (black, Ortho); $\boldsymbol{E}, 0.89 \pm 0.02$ (red, Sub wK $_{\text {) }}, 0.84 \pm 0.02$ (black, 0rtho), $0.83 \pm 0.02$ (blue, 0pt); $\boldsymbol{F}, 1.01 \pm 0.04$ (black, 0rtho), $0.94 \pm 0.02$ (green, Sub), $0.88 \pm 0.02$ (blue, 0pt). All means in $\boldsymbol{D}-\boldsymbol{F}$ are significantly different from $1\left(^{*} p<0.05\right.$ and $^{* *} p<0.001$, Student's $t$ test), except for the black arrow in $\boldsymbol{F}$.

In Figure $4 A$, for each center orientation, we plot the normalized response averaged across the population to a center stimulus presented alone (hatched bars) or together with a surround stimulus at each of the four different orientations (solid bars). Again, the iso-orientation condition was significantly more suppressive than the non-iso-orientation conditions $(p<0.001, p<0.001$, and $p=0.017$ for Opt, Sub, and Sub $\mathrm{WK}_{\mathrm{WK}}$ center orientations, respectively, ANOVA with contrasts), and surround stimulus ori- 


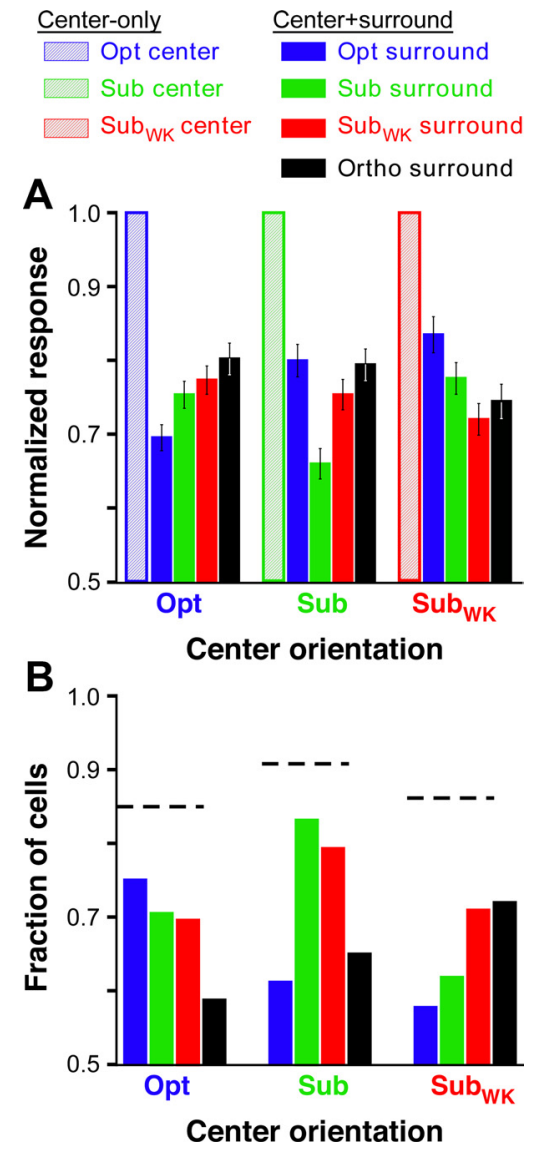

Figure 4. Orientation tuning of surround suppression: population data. A, Average normalized population responses (calculated as described in the legend to Fig. $3 A-C$ ). Each set of five bars indicates the mean normalized response to a given center stimulus orientation (indicated on the $x$-axis) presented alone (hatched bar) or together with a surround stimulus presented at each of four different orientations (solid bars color coded as in the legend at the top). $\boldsymbol{B}$, Each set of four bars indicates, for each center orientation (indicated on the $x$-axis), the fraction of cells showing significant suppression at each of four different surround orientations. Horizontal dashed lines, Total fraction of cells, for each center stimulus orientation, suppressed by at least one surround orientation. Legend at the top applies to both $\boldsymbol{A}$ and $\boldsymbol{B}$.

entations nearest to that in the RF caused stronger suppression than surround stimulus orientations nearest-to-orthogonal to that in the RF. Furthermore, more cells showed suppression in the iso-orientation condition than in the non-iso-orientation conditions. This is shown in Figure $4 B$ where, for each center orientation, we plot the fraction of cells showing significant suppression at each surround stimulus orientation (significant suppression was defined as a neuronal response in the presence of a surround stimulus that was at least 1 SEM below the center-only response at the respective orientation). The dashed horizontal lines indicate the fraction of cells at each center orientation for which at least one of the surround orientations was significantly suppressive. Although the total fraction of cells showing suppression was similar across different center stimulus orientations, the proportion of cells showing suppression at iso-orientations versus non-iso-orientations varied. Specifically, a larger fraction of cells showed suppression when the surround stimulus orientation matched or approached that in the RF, whereas a smaller fraction of neurons showed suppression for surround stimulus orientations increasingly different from the center stimulus orientation. Notice that, in our conventions, a surround stimulus of Opt orientation is nearest-to-orthogonal to a center stimulus of $\mathrm{Sub}_{\mathrm{WK}}$ orientation (Fig. $4 B$, rightmost set of bars).
Facilitatory surround effects and their orientation dependence

To investigate the orientation dependence of surround facilitatory effects, we analyzed neuronal responses to smaller surround stimuli (i.e., surround annuli with larger inner diameter) than those used for our analysis of suppression. This is because, in previous studies, we found that surround facilitation is typically observed when both the RF and surround are weakly stimulated, as for example, when the RF center is stimulated with a low contrast grating and the surround with a thin annular grating (Ichida et al., 2007). Large surround stimuli, even when the RF center was weakly stimulated, typically evoked suppression. Indeed, in Figure $3 A-C$, the larger surround stimuli used for the analysis caused predominantly suppressive effects, regardless of the orientation at which they were presented or of the center stimulus orientation. Only few cells were facilitated by these larger surround stimuli (i.e., cells with normalized response $>1$ in Fig. $3 A-C$ ).

Therefore, to assess the orientation dependence of surround facilitation, we analyzed the modulatory effects caused by thin surround annuli located far from the RF center. However, because different cells have different far surround sizes (Ichida et al., 2007), we could not perform this analysis at the same surround stimulus size for all cells. This is because any given surround annulus size could cause suppression in one set of cells, facilitation in a different set of cells, and no modulatory effect in yet other set of cells. Thus, our analysis of facilitation was performed for each cell at the surround size (inner diameter) that evoked the largest response from the cell, regardless of the specific combination of center-surround stimulus orientations that evoked such response.

Figure 5, $A$ and $B$, shows the response of an example cell to center and surround gratings presented at all combinations of four orientations. The area of each square in $B$ corresponds to the response of the cell to that particular combination of center and surround orientations, at the surround size that evoked strongest facilitation (in any center-surround orientation condition), normalized to the center-only response at the Opt orientation. Strong facilitation occurred when the RF was stimulated with suboptimal (Sub and $\mathrm{Sub}_{\mathrm{WK}}$ ), but not with Opt, orientations, and it was maximal when the surround stimulus orientation was nearest-to-orthogonal to the stimulus orientation in the RF (marked by an asterisk). Weakest facilitation occurred in the isoorientation conditions. Furthermore, a center stimulus of Ortho orientation evoked no significant response from the cell, with or without a surround stimulus of any orientation, and this was the case across our cell sample. In other words, the surround stimulus had no significant effect on the spiking response of a cell, when the cell was not responding to the center stimulus alone. This observation is inconsistent with a previous report (Sillito et al., 1995) (see Discussion). Figure 5C plots the response of the same cell in a different format, i.e., as normalized response as a function of surround stimulus orientation. Colored curves correspond to different center stimulus orientations, and the cell response was normalized to the center-only response at the corresponding orientation. Again, facilitation occurred only when the center was stimulated with suboptimal orientations for the cell, and strongest facilitation occurred for surround orientations nearest-to-orthogonal to the center stimulus orientation (marked by an asterisk in Fig. 5C).

The population data for surround facilitation are shown in Figures 6 and 7. Figure $6 A-C$ shows, for each of three different center stimulus orientations, scatter plots of the normalized response (measured as in Fig. 5C) in the iso-orientation condition versus the response in the three non-iso-orientation conditions. 

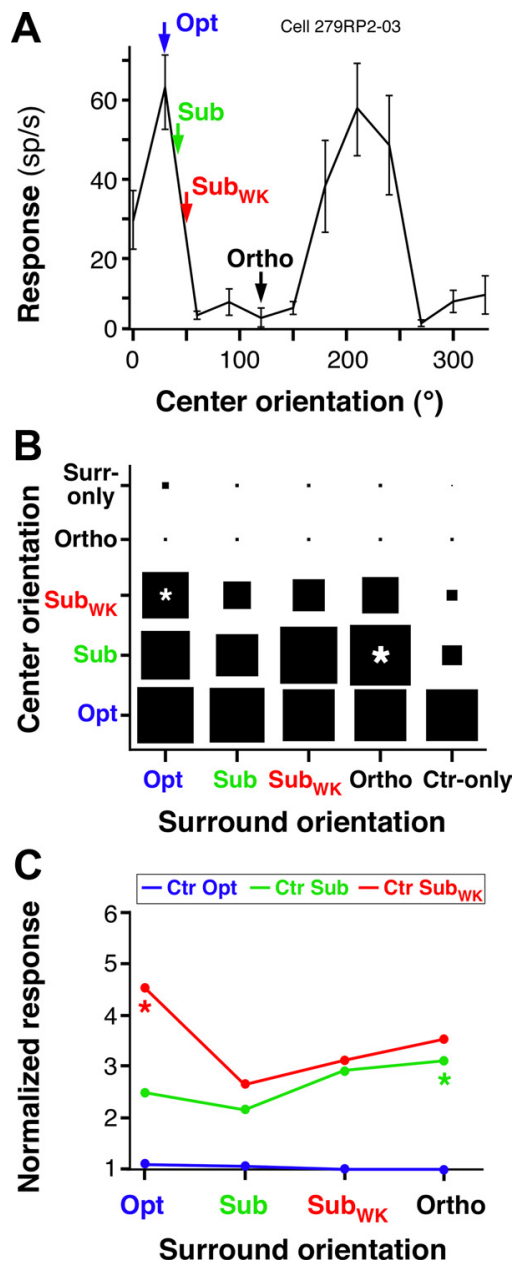

Figure 5. Response of one example cell to center and small surround gratings of changing orientation. $\boldsymbol{A}$, Orientation tuning curve of the RF response for an example V1 cell showing surround facilitation. The arrows indicate the four orientations chosen for this cell to characterize the orientation specificity of surround facilitation. $\boldsymbol{B}$, Response of the same example cell to center and surround gratings presented at all combinations of the four orientations indicated by arrows in $\boldsymbol{A}$. All responses are normalized to the center-only response at the 0 pt orientation. $\boldsymbol{C}$, For each of three different center orientations, indicated as curves of different colors, we show the response of the same cell as in $\boldsymbol{A}$ and $\boldsymbol{B}$ to center-surround stimuli, as a function of surround stimulus orientation. Here responses are normalized to the center-only response at each respective orientation. Asterisks in $\boldsymbol{B}$ and $\boldsymbol{C}$ mark the center-surround orientation condition evoking the largest facilitation. Other conventions are as in Figure 2.

Compared with Figure $3 A-C$, here many more cells have normalized responses $>1$ at both iso-orientations and non-iso orientations, i.e., showed surround facilitation. The percentage of facilitating cells was greater when the RF center was weakly stimulated with $\mathrm{Sub}_{\mathrm{WK}}$ orientations than when it was strongly stimulated by a stimulus of Opt or Sub orientations. Furthermore, for any given center orientation, the percentage of cells showing facilitation was greater in the non-iso-orientation conditions than in the iso-orientation condition. Specifically, for the Opt center orientation (Fig. 6A), 27\% of cells were facilitated by an isooriented surround stimulus versus 33,35 , and $43 \%$ by a Sub, $\mathrm{Sub}_{\mathrm{WK}}$, and Ortho surround stimulus orientation, respectively. For the Sub center orientation (Fig. 6B), 18\% of cells were facilitated in the iso-orientation condition versus $37 \%(\mathrm{Opt}), 29 \%\left(\mathrm{Sub}_{\mathrm{WK}}\right)$, and $40 \%$ (Ortho) in each non-iso-orientation condition. For the Sub $_{\mathrm{WK}}$ center orientation (Fig. 6C), 32\% of cells were facilitated in the iso-orientation condition versus $48 \%$ (Opt), 42\% (Sub), and $41 \%$ (Ortho) in each non-iso-orientation condition. Furthermore, in each panel of Figure $6 A-C$, most points lie above the diagonal, i.e., for most cells, the response at non-iso-orientation was greater than at iso-orientation. This indicates that, for most cells, the isoorientation condition was the least facilitatory (for cells with response $>1$ ) or the most suppressive (for cells with response $<1$ ). However, even at the surround sizes optimized to reveal facilitatory surround effects, the majority of cells did not show facilitation, even in the $\mathrm{Sub}_{\mathrm{Wk}}$ center condition; rather they showed suppression or no surround effects. This indicates that suppression is the dominant surround effect, at least at the stimulus contrast and orientations used in this study.

Figure $6 D-F$ shows the distribution of responses to different surround stimulus orientations for each center orientation condition. The response ratio (response at iso-orientation/response at non-iso-orientation) was $<1$ for the majority of cells at most surround orientations, indicating that responses are lowest, i.e., facilitation is weakest, for iso-oriented center-surround stimuli. This was independent of center stimulus orientation. All mean ratios (arrows) in Figure 6, D and $E$, were highly significantly different from 1; in Figure $6 F$, instead, only the surround orientation (Opt) that was nearest-to-orthogonal to that in the RF $\left(\mathrm{Sub}_{\mathrm{WK}}\right)$ caused a significantly greater response than an isooriented surround stimulus.

Figure $6 D-F$ also shows that surround stimulus orientations nearest the orientation of the stimulus in the RF evoked weaker responses (i.e., weaker facilitation) than surround orientations nearest-to-orthogonal to the orientation in the RF. Thus, for example in Figure $6 D$, in which the center stimulus was at Opt, Sub stimuli in the surround (green bars) evoked weaker facilitation than Ortho surround stimuli (black bars); however, the mean ratios between these two surround conditions did not differ significantly $(p=0.057)$. Similarly, in Figure $6 F$, in which the center stimulus was at $\mathrm{Sub}_{\mathrm{WK}}$, Ortho surround stimuli (which are closest in orientation to $S u b_{\mathrm{WK}}$ ) evoked weaker responses (i.e., weaker facilitation) than Opt surround stimuli (which are nearest-to-orthogonal to $\mathrm{Sub}_{\mathrm{WK}}$ ), but again the mean ratios for these two surround orientations were not statistically different $(p=0.12)$. Note, however, that the distribution of response ratios in Figure 6D-F included all cells in our sample, i.e., cells that showed surround facilitation, as well as cells that showed surround suppression or no surround effects, under the same stimulus conditions.

Thus, to better characterize the orientation dependence of surround facilitation, we selected from our neuronal population only the cells that showed surround facilitatory effects. The orientation tuning of surround facilitation for this population is shown in Figure 7A. Here, for each center stimulus orientation, we plot the mean normalized population response at each of four different surround stimulus orientations. Facilitation was significantly weaker in the iso-orientation condition, regardless of center stimulus orientation $(<0.003,<0.005$, and $<0.02$ for Opt, Sub, and Sub $\mathrm{WK}_{\mathrm{WK}}$ center orientations, respectively, ANOVA with contrasts). Furthermore, the surround orientation nearest-toorthogonal to the stimulus orientation presented in the RF caused significantly stronger facilitation than surround orientations nearest to the center stimulus orientation. Thus, in the Opt center condition (Fig. 7A), Sub stimuli in the surround (green bars) evoked weaker facilitation than Ortho surround stimuli, and this difference in response was statistically significant $(p=$ 0.012 , paired Student's $t$ test). Instead, in the Sub ${ }_{\mathrm{WK}}$ center condition, surround stimuli of Sub orientation evoked significantly $(p=0.013$ ) weaker facilitation than Opt surround stimuli (whose orientation is the nearest-to-orthogonal to $\mathrm{Sub}_{\mathrm{WK}}$ ). 


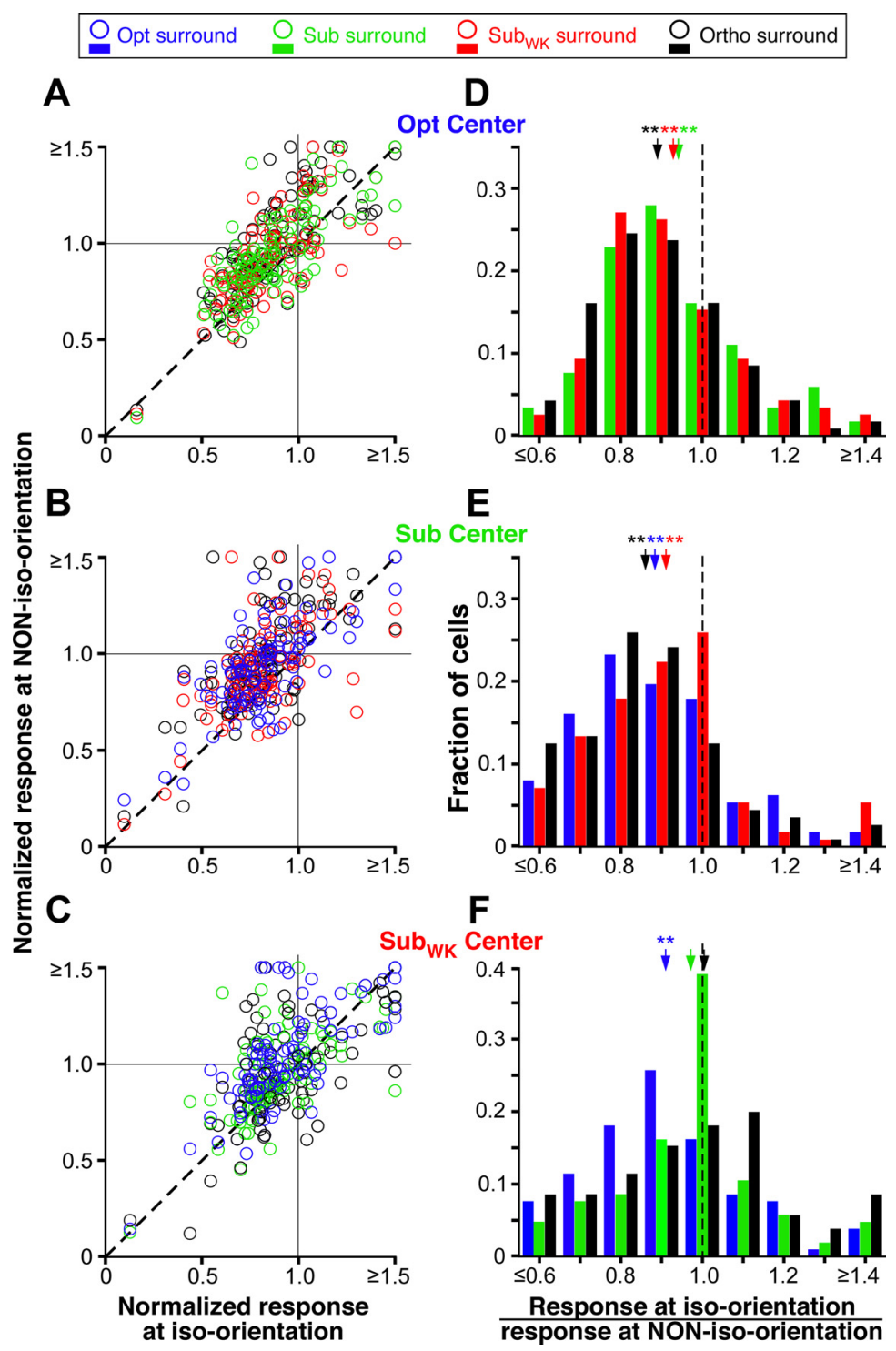

Figure 6. Orientation specificity of surround facilitation: population data. $A-C$, Scatter plots of normalized responses to isooriented center-surround stimuli versus responses to each of three non-iso-oriented center-surround stimuli. Each panel shows responses to a different center stimulus orientation (indicated at the top right corner of each panel). The normalized response was estimated as the response to a particular combination of center and surround stimulus orientation, at the surround size evoking strongest facilitation in any orientation condition, and normalized to the center-only response at each respective orientation. Other conventions are as in Figure $3 A-C . \boldsymbol{D}-\boldsymbol{F}$, Each panel shows, for a different center stimulus orientation, the distribution of responses to three surround stimulus orientations responses calculated as in Figure 3, $\mathbf{D}-\boldsymbol{F}$, and color coded as per legend at the top of the figure). Mean ratios (arrows) \pm SEM are as follows: $\boldsymbol{D}, 0.94 \pm 0.02$ (green, Sub), $0.93 \pm 0.02$ (red, Sub wk $_{\text {) }}$ ) $0.89 \pm 0.01$ (black, Ortho); $\boldsymbol{E}, 0.91 \pm 0.02$ (red, Sub $\left.{ }_{w k}\right), 0.88 \pm 0.02$ (blue, 0 pt), $0.86 \pm 0.02$ (black, Ortho); $\boldsymbol{F}, 1.0 \pm 0.03$ (black, Ortho), $0.97 \pm 0.02$ (green, Sub), $0.91 \pm 0.02$ (blue, 0pt). All means in $\boldsymbol{D}$ and $\boldsymbol{E}$ and the $0 \mathrm{pt}$ (blue) surround condition in $\boldsymbol{F}$ are significantly different from $1\left({ }^{*} p<0.05\right.$ and ${ }^{* *} p<0.001$, Student's $t$ test). Other conventions are as in Figure $3 D-F$.

In Figure $7 B$, for each center stimulus orientation, we plot the fraction of cells showing significant surround facilitation at each surround orientation (significant facilitation was defined as a response to a center-surround stimulus $>1$ SEM above the response to the center-only stimulus at the respective orientation). The horizontal lines indicate the total fraction of cells at each center orientation showing significant facilitation for at least one surround orientation. A larger fraction of cells (35\%) showed significant facilitation in the $\mathrm{Sub}_{\mathrm{WK}}$ center condition compared with the Opt or Sub center conditions. Furthermore, for any given center orientation, a smaller fraction of cells showed facilitation when the surround orientation matched that in the RF center, whereas the largest fraction of facilitating cells was seen for surround stimuli oriented orthogonal or nearest-to-orthogonal to the orientation of the center stimulus.

\section{Computation of orientation-tuned surround suppression: a recurrent network model}

What mechanism could adjust the orientation specificity of the surround to the stimulus presented to the RF? To understand the possible mechanisms underlying these experimental results, we explored the behavior of a simple recurrent network model of V1.

We modeled a cortical orientation hypercolumn as 32 recurrently connected orientation columns. Each orientation column consisted of three neuronal populations, one excitatory (E) and two inhibitory (I and B) (Fig. 8A, top). $\mathrm{I}$ is a high-threshold local inhibitory neuron (Somers et al., 1998; Bressloff and Cowan, 2002; Schwabe et al., 2006) that projects only to E neurons within the same orientation column and that serves to relay horizontal- and feedback-mediated suppression from the surround. We (Schwabe et al., 2006) and others (Somers et al., 1998) showed previously that highthreshold I neurons allow to reproduce the contrast dependence of surround modulation (Sceniak et al., 1999; Schwabe et al., 2010), as well as facilitation from the far surround when the RF and surround are weakly stimulated (Ichida et al., 2007). B is a basket-like inhibitory neuron that connects with $\mathrm{E}$ cells within the same and other orientation columns (Lund and Yoshioka, 1991; Kisvárday et al., 1994; Lund et al., 1995; Lund and Wu, 1997; Burkhalter, 2008).

$\mathrm{E}$ and $\mathrm{B}$ make recurrent connections onto themselves, as well as reciprocal connections onto each other both within the same column and between different orientation columns (Fig. 8A; for simplicity, we only depict two orientation columns). $\mathrm{E}$ and $\mathrm{B}$ cells in different orientation columns are also recurrently connected with other E and B cells, respectively. Importantly, these recurrent connections between orientation columns are strong, with strong excitation balanced by strong inhibition (Bressloff and Cowan, 2002; Mariño et al., 2005; Stimberg et al., 2009).

$\mathrm{E}$ and $\mathrm{B}$ cells in each orientation column receive feedforward inputs in the RF center from other cortical layers (layer 4C). Other external inputs arise from the surround and preferentially activate the I and B cells, via excitatory V1 HCs and inter-areal FB connections (Angelucci et al., 2002). Following anatomical evidence, in the model the latter two connection types are orientation specific (Malach et al., 1993; Shmuel et al., 2005) and target both excitatory and inhibitory neurons (McGuire et al., 1991; 

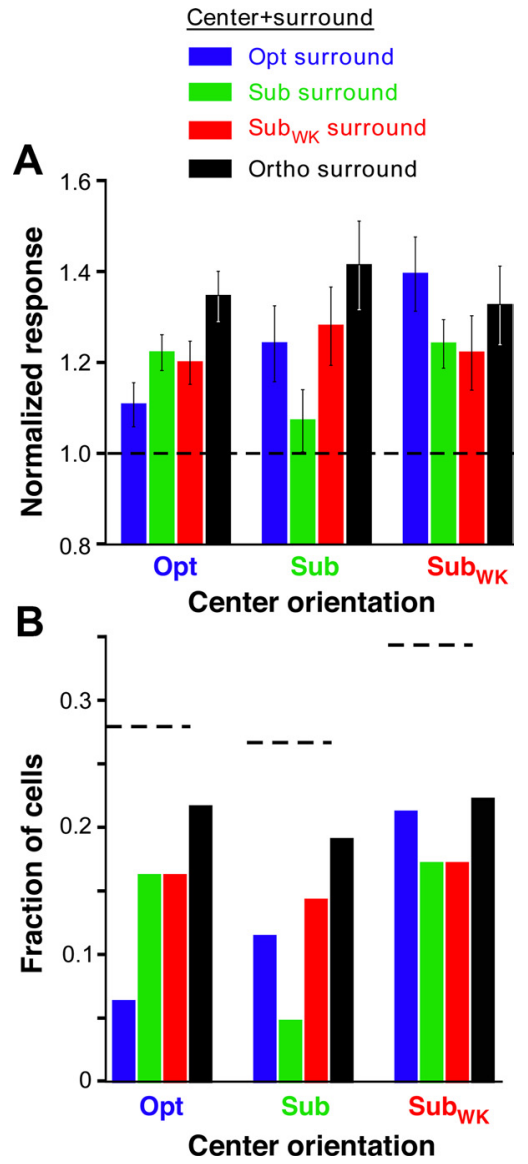

Figure 7. Orientation tuning of surround facilitation: population data. A, Average normalized population responses for cells showing surround facilitation. Each set of four bars indicates the mean normalized response to a given center stimulus orientation presented together with a surround stimulus at each of four different orientations. $\boldsymbol{B}$, Each set of four bars indicates, for each center orientation, the fraction of cells showing significant facilitation at each of four different surround orientations. Horizontal dashed lines, Total fraction of cells, for each center stimulus orientation, that were facilitated by at least one surround orientation. Other conventions are as in Figure 4.

Gonchar and Burkhalter, 2003; Anderson and Martin, 2009). In contrast, local recurrent connections, by virtue of their local connectivity with all orientation columns, are poorly orientation tuned.

This model architecture closely resembles that of our previously described model of horizontal- and feedback-mediated surround modulation (Schwabe et al., 2006), but it extends it in two important ways. First, it explicitly incorporates the orientation tuning of RF center responses and surround modulation. Second, it incorporates strong local recurrent connections between orientation columns. We show that this model can reproduce the experimental data on the orientation dependence of surround suppression (Fig. 8A, bottom) and that this results from the interaction of orientation-specific surround inputs (via $\mathrm{HCs}$ and $\mathrm{FB}$ connections) with strong and only weakly orientation-specific local recurrent connections (Fig. 9).

Figure $8 \mathrm{~A}$ shows the simulated response of the model of the $\mathrm{E}_{1}$ neuron with $0^{\circ}$ preferred (Opt) orientation, for varying surround stimulus orientations, with the center stimulus presented either at the Opt (solid curve) or at the Sub $\left(22.5^{\circ}\right.$, dashed curve) orientation. Surround suppression is strongest when center and surround stimuli are at the same orientation, even when the center stimulus is at Sub orientation. Importantly, only if V1 operates in a regime of strong local recurrence is the suppression tuned to the stimulus presented to the RF center. In Figure $8 \mathrm{~B}$, we show that, in a model with no recurrent connections between orientation columns, surround suppression is always tuned to the preferred orientation $\left(0^{\circ}\right)$ of the E neuron. Specifically, we used a different parameterization of the network model, in which orientationtuned surround suppression is mediated exclusively via orientation-specific surround pathways (HCs and FB connections) acting via the I neuron (Fig. $8 \mathrm{~B}$, top). Using the parameterization of this model, we simulated the $\mathrm{E}_{1}$ neuron response to surround stimuli of varying orientation, with the center stimulus orientation set at Opt $\left(0^{\circ}\right)$ or at $\mathrm{Sub}\left(22.5^{\circ}\right)$ (Fig. $8 B$, bottom). In this case, strongest suppression occurs when the surround stimulus is at the Opt orientation for the $\mathrm{E}_{1}$ cell, even when its RF center is stimulated with a Sub orientation. In Figure $8 C$, we show the performance of a previously proposed model of surround suppression (Ozeki et al., 2009), in which strong recurrence exists only between excitatory and inhibitory neurons within an orientation column. This model predicts the same orientation tuning of surround suppression as the model in Figure $8 \mathrm{~B}$. Thus, strong local recurrent connections within an orientation column are not sufficient to predict the experimentally observed iso-orientation suppression independent of the preferred orientation of the cell. That is, strong recurrence between orientation columns is needed to account for the experimental results presented in Figures 2-7.

The underlying mechanism in the recurrent model is illustrated in Figure 9. In Figure 9, $A$ and $D$, we show the inputs into the model $\mathrm{E}_{1}$ neuron, preferring $0^{\circ}$ orientation, for varying surround orientations, when the center is stimulated with an Opt $\left(0^{\circ} ; A\right)$ or with a Sub $\left(22.5^{\circ} ; D\right)$ orientation. It is the net sum of local recurrent (both excitatory and inhibitory), horizontal and feedback inputs that determines the $\mathrm{E}_{1}$ neuron response to center-surround stimuli, i.e., the response shown in Figure $8 \mathrm{~A}$. When the RF center and surround are stimulated with an $\mathrm{Opt}$ orientation (Fig. 9A, arrow), inhibition via the surround pathways is strongest and recurrent excitation is weakest. However, when the center and surround stimuli are at a Sub orientation (Fig. 9D, arrow), although the inhibition via the surround is weaker, recurrent excitation is weakest; this withdrawal of recurrent excitation exceeds the reduction in surround inhibition and causes suppression at the feedforward input orientation. Why is this? The diagrams in Figure 9 illustrate the underlying mechanism. For simplicity of illustration, only the inputs and neuron types that most affect the $\mathrm{E}_{1}$ cell response are shown in these diagrams; thus, weak inputs are omitted, and the surround pathways are shown to activate only the I cells. B neurons are also omitted for simplicity because their role, albeit important, is simply to balance the strong recurrent excitation (recurrent inhibition follows the tuning of recurrent excitation; Fig. $9 A, D)$. When the center is at the Opt orientation for the $\mathrm{E}_{1}$ cell (Fig. $9 A-C$ ), the $\mathrm{E}_{1}$ cell receives strongest feedforward activation (green line in $B$, $C$ ) and thus provides the strongest recurrent excitation within the hypercolumn (thick blue lines in $B$ ). Because of the strong recurrent regime within the hypercolumn, the whole hypercolumn becomes strongly activated. When the $\mathrm{E}_{1}$ cells are suppressed by an Opt surround stimulus (via HCs and FB connections; red line in $C$ ), the strongest source of recurrent excitation within the hypercolumn, i.e., the $\mathrm{E}_{1}$ cell population, is suppressed; recurrent excitation is, thus, withdrawn (thin blue lines in $C$ ) and the whole hypercolumn becomes suppressed. This withdrawal of excitation is greater when the $\mathrm{E}_{1}$ cell is maximally suppressed by an $\mathrm{Opt}\left(0^{\circ}\right)$ surround stimulus $(C)$, than when it is weakly suppressed or not suppressed by a Sub $\left(22.5^{\circ}\right)$ or other non-optimal surround stim- 
A

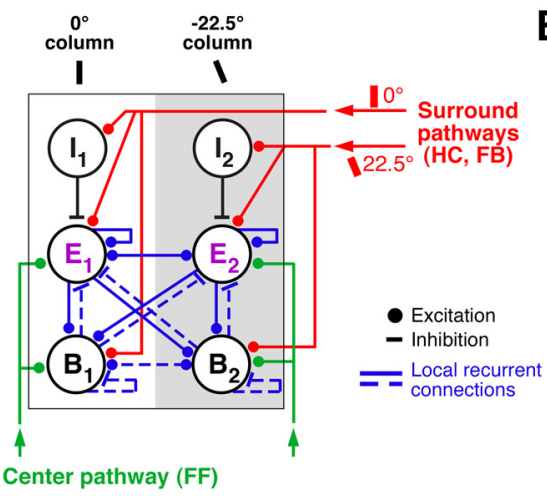

B

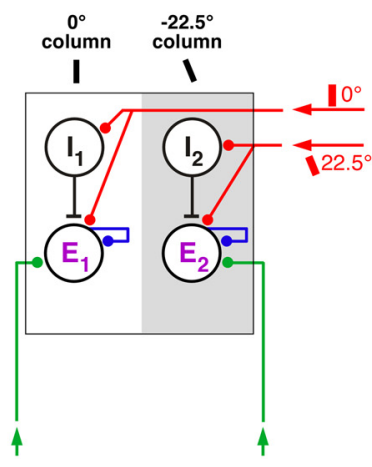

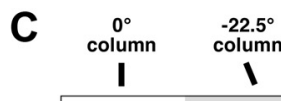

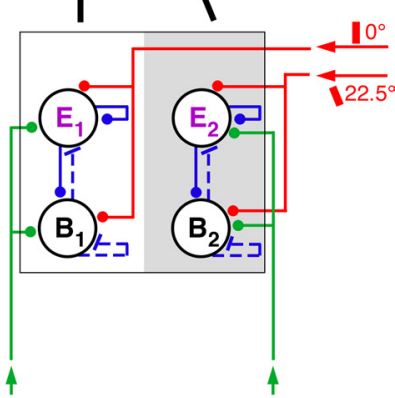

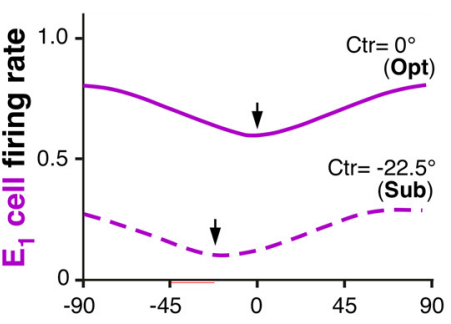
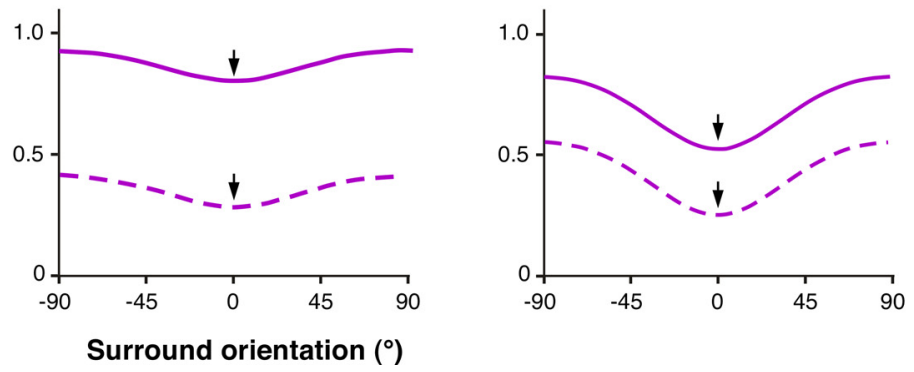

Figure 8. Orientation dependence of surround suppression in the model. $A$, Tuning of suppression in the model with strong local recurrence. Top, The recurrent network model architecture. $I_{1}, E_{1}, B_{1}$, Three population of cells, i.e., local inhibitory, excitatory, and basket, respectively, located in the same $0^{\circ}$-preferring orientation column (white) within the center hypercolumn. $I_{2}, E_{2}, B_{2}$, The same three cell types located in a different column, i.e., one preferring $22.5^{\circ}$ orientation (gray). For simplicity of illustration, only two orientation columns are shown, but in the model, the center hypercolumn consists of 32 recurrently connected orientation columns. Excitatory feedforward inputs driven by the stimulus in the RF center and excitatory orientation-specific inputs driven by the stimulus in the surround are indicated in green and red, respectively, whereas blue indicates local recurrent connections within and between orientation columns. Bottom, Simulated response of the $\mathrm{E}_{1}$ cell, with preferred orientation $0^{\circ}$, for varying surround stimulus orientations, with the stimulus in the RF presented at $0^{\circ}$ (Opt orientation, solid curve) or at $22.5^{\circ}$ (Sub orientation, dashed curve). Arrows point at the surround orientation that caused maximal suppression. $\boldsymbol{B}$, Tuning of suppression in the model with no strong recurrent connections within and between orientation columns. Top, The model architecture. Again, for simplicity only two orientation columns are shown $\left(0^{\circ}\right.$ and $22.5^{\circ}$-preferring columns). Excitatory and orientation-specific surround inputs (red) contact both I and E neurons. Feedforward inputs (green) contact only E neurons. I is the same high-threshold local inhibitory neuron of the model in $A$. Bottom, Simulated response of the $E_{1}$ neuron with preferred orientation $0^{\circ}$ to surround stimuli of varying orientation, with the center stimulus set at $\mathrm{Opt}\left(0^{\circ}\right)$ or Sub $\left(22.5^{\circ}\right)$. C, Tuning of suppression in a model with strong local recurrent connections existing only within orientation columns. Top, The model architecture. E, B, Populations of excitatory and inhibitory neurons, respectively. The inhibitory neuron population resembles the $B$ neuron population of the model in $\boldsymbol{A}$ (i.e., like $B$ in the model shown in $\boldsymbol{A}$, it serves to balance the strong recurrent excitation). Excitatory feedforward (green) and surround inputs (red) contact both $B$ and $E$ neurons. Strong recurrent connections (blue) exist only within columns but not between different orientation columns. Bottom, Simulated response of the E1 neurons in this model.

ulus, respectively $(B)$. The Sub surround stimulus (red line in $B$ ), instead, maximally suppresses the $\mathrm{E}_{2}$ cell in the adjacent orientation column preferring $22.5^{\circ}$. However, because the $\mathrm{E}_{2}$ cell is less activated by the $0^{\circ}$ center stimulus compared with the $\mathrm{E}_{1}$ cell, it provides weak recurrent excitation to the rest of the hypercolumn; thus, suppressing $\mathrm{E}_{2}(B)$ results in lesser withdrawal of excitation within the hypercolumn than suppressing the more active $\mathrm{E}_{1}$ cell $(C)$.

Let us now examine the condition when the RF center is stimulated suboptimally (Fig. $9 D-F$ ). In this case, the $\mathrm{E}_{1}$ cell receives weaker feedforward activation $(E, F)$, and therefore provides weaker recurrent excitation to the hypercolumn compared with the previous scenario. Thus, suppression of the $\mathrm{E}_{1}$ cell (red line in $E$ ) by a $0^{\circ}$ surround stimulus (i.e., Opt for the $\mathrm{E}_{1}$ cell) has little effect on the overall hypercolumn activity. However, the $\mathrm{E}_{2}$ cell, which is maximally activated by the Sub $\left(22.5^{\circ}\right)$ center stimulus (green line in $E, F$ ), provides the strongest recurrent excitation to the hypercolumn (thick blue lines in $E$ ) and thus to the $\mathrm{E}_{1}$ cell. When the $\mathrm{E}_{2}$ cell is maximally suppressed by a surround stimulus of $22.5^{\circ}$ orientation (i.e., Sub for the $\mathrm{E}_{1}$ cell but $\mathrm{Opt}$ for the $\mathrm{E}_{2}$ cell; red line in $F$ ), the strongest recurrent excitation to the whole hypercolumn, and thus to the $\mathrm{E}_{1}$ cell, is withdrawn (thin blue lines in $F$ ). Instead, a surround stimulus of $0^{\circ}$ (Sub for the $\mathrm{E}_{2}$ cell) does not suppress or only weakly suppresses the $\mathrm{E}_{2}$ cell (red line in
$E)$, resulting in little or no withdrawal of recurrent excitation to the $\mathrm{E}_{1}$ cell (thick blue line in $E$ ). In summary, recurrent excitation is weakest when the surround stimulus is of the same orientation as the center stimulus $(C, F)$; this is because, in this stimulus configuration, the orientation-specific surround pathways maximally suppress the center orientation columns that are tuned to, and therefore most activated by, the center stimulus orientation and which thus provide the strongest recurrent excitation to the whole hypercolumn. Because of the strong recurrent regimen, the level of recurrent excitation in the hypercolumn has a greater effect on the $\mathrm{E}_{1}$ neuron activity than the direct inhibition via the surround pathways. Thus, because recurrent excitation is minimal at iso-orientation, regardless of the stimulus orientation in the RF center, surround suppression shows the same tuning behavior. Conversely, the local recurrent connections by virtue of their connectivity are not orientation specific. Functional tuning of withdrawal of recurrent excitation to the stimulus orientation in the RF thus results from the interaction of orientation-specific surround inhibition, through the surround pathways, with orientation-unspecific local recurrent connections. In summary, the orientation tuning of surround suppression results from the interactions of two differently tuned mechanisms, a tuned surround inhibitory mechanism and an untuned center excitatory 


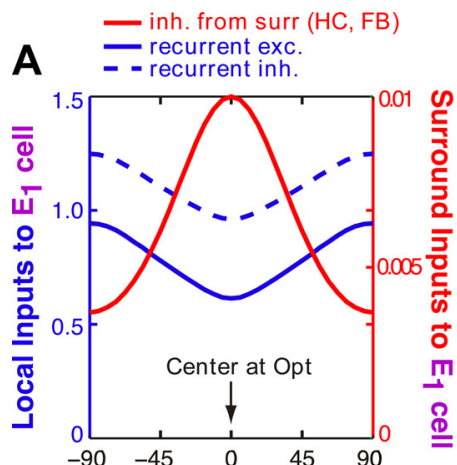

Surround orientation $\left({ }^{\circ}\right)$

D

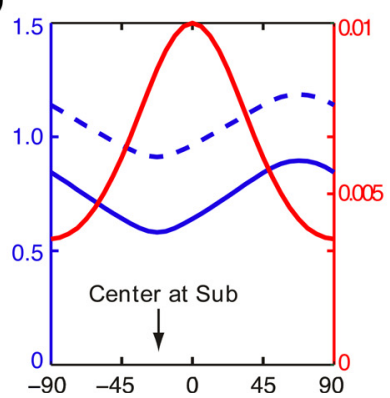

B

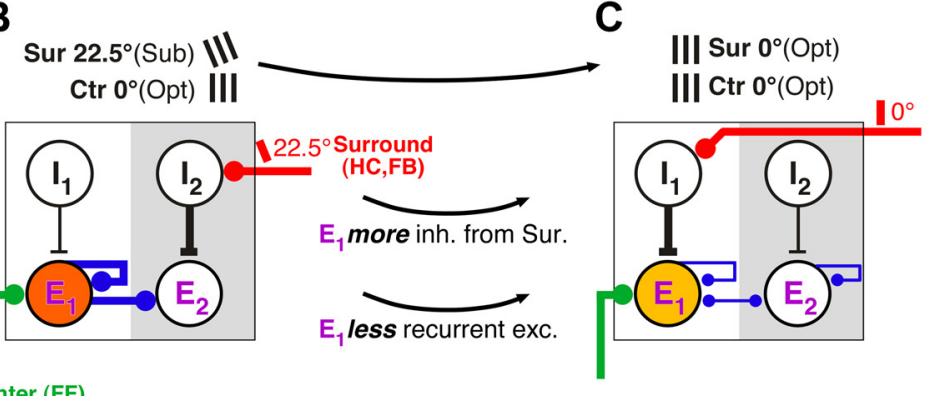

$E_{1}$ cell response

E

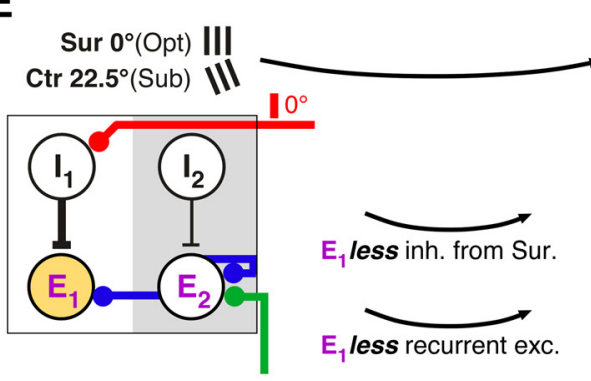

III Sur22.5 (Sub) III Ctr $22.5^{\circ}$ (Sub)

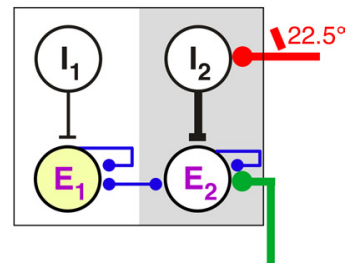

Figure 9. Dependence of surround suppression on the stimulus orientation presented to the RF: underlying mechanism in the recurrent model. $\boldsymbol{A}$, Inputs into the $E_{1}$ model cell preferring $0^{\circ}$ orientation, for varying surround orientations, when its $\mathrm{RF}$ center is stimulated with a $0^{\circ}$, i.e., 0 pt, orientation (corresponding to the purple solid line in Fig. $4 \mathrm{~A}$ ). Solid blue curve, Local recurrent inputs from E neurons in other orientation columns (e.g., $E_{2}$ ); dashed blue curve, Local recurrent inputs (negative) from basket neurons in the same and other orientation columns; red curve, input (negative) from the surround. Note that the surround input (red $y$-axis) is almost two orders of magnitude smaller than the local recurrent inputs (blue $y$-axis). $B, C$, The diagrams illustrate the inputs that most affect the $E_{1}$ cell response, when the center stimulus is at the Opt orientation for the $E_{1}$ cell and the surround is either at the Sub $\left(22.5^{\circ} ; \boldsymbol{B}\right)$ or at the $0 \mathrm{pt}\left(0^{\circ} ; \boldsymbol{C}\right)$ orientation for the $\mathrm{E}_{1}$ cell. Line thickness indicates input strength. For simplicity of illustration, only the I and $E$ neurons are shown within each column (white is the $0^{\circ}$ and gray the $22.5^{\circ}$ orientation column), and the surround pathways are assumed to activate only the I cells. Changing the surround stimulus orientation (top black arrow) from the Sub to the iso-orientation condition (Opt center and surround) leads to increased inhibition of the $E_{1}$ cell via the surround inputs and to less recurrent excitation. $\boldsymbol{D}$, Same as in $\boldsymbol{A}$ but for a center stimulus orientation of $-22.5^{\circ}$ (corresponding to the purple dashed line in Fig. $4 A$ ). $\boldsymbol{E}, \boldsymbol{F}$, Here, the diagram illustrates that changing the surround stimulus orientation (top black arrow) from the $\mathrm{Opt}$ to the iso-orientation condition (Sub center and surround) leads to reduced inhibition of the $\mathrm{E}_{1}$ cell via the surround pathways but also to less recurrent excitation. The reduced recurrent excitation is what causes stronger suppression at iso-orientation.

mechanism (note that local recurrent inhibition follows the tuning of the recurrent excitation; Fig. $9 A, D$, dashed curve).

\section{Discussion}

We found that, for most V1 cells, surround modulation is independent of the RF tuning of the individual neuron; instead, it depends on the stimulus orientation presented to the RF. Suppression is maximal at iso-orientation and facilitation at crossorientation, even when the stimulus in the RF is not at the preferred orientation of the cell, provided this stimulus can reliably activate the cell when presented without a surround stimulus. This result cannot be explained solely by horizontal and/or FB connections with fixed orientation specificities. Instead, we have shown that this tuning can emerge only if V1 operates in a regime of strong local recurrence, and it results from the interaction of two differently tuned intracortical mechanisms: (1) lateral inhibition, via HCs and/or FB connections, which is tuned to the preferred orientation of the cell, and (2) withdrawal of recurrent excitation, via weakly tuned local recurrent connections, which becomes tuned to the feedforward input orientation as a result of tuned lateral inhibition.

Thus, contextual modulation of strong local recurrent circuits by HCs and FB connections can support complex computations, allowing the tuning of neuronal responses to change dynamically with changing feedforward inputs.
The orientation specificity of surround suppression and facilitation is independent of the RF orientation specificity Most previous studies on the orientation tuning of surround modulation in V1 have suggested that this is generated intracortically by orientation-specific HCs and FB connections. The timing of this modulation (Smith et al., 2006), its spatial extent (Sceniak et al., 2001; Angelucci et al., 2002; Cavanaugh et al., 2002a; Levitt and Lund, 2002), and the lack of tuned suppression in the LGN (Solomon et al., 2002; Webb et al., 2002) also suggest a cortical origin of surround modulation. This idea was challenged by reports (Sillito et al., 1995; Cavanaugh et al., 2002b) that, in some V1 cells, the orientation tuning of surround modulation is not fixed but changes with the stimulus orientation in the RF. This tuning cannot be generated solely by HCs and FB connections, because these have fixed orientation specificity. However, the prevalence of this phenomenon in V1 remained unclear. This is because, in one study (Sillito et al., 1995), this effect was shown in only four V1 cells, and it was not quantified across the population, whereas in the second study (Cavanaugh et al., 2002b), this effect was replicated in approximately half of a population of 27 broadly orientation-tuned cells and only for suppressive surround effects. Here we have demonstrated that strongest suppression at iso-orientation independent of the preferred orientation of the individual cells is highly prevalent in V1, including sharply orientation-tuned cells. 
There is disagreement regarding the occurrence and orientation tuning of surround facilitation. Sillito et al. (1995) reported that any orientation discontinuity of stimuli in the RF and surround evoked facilitation, even when the RF center was stimulated by an orthogonal-to-optimal orientation, which does not activate the cell in the absence of surround stimulation. Cavanaugh et al. (2002b), instead, did not find any consistent facilitatory effects and argued that facilitation could result from encroachment of the surround stimulus on the subthreshold excitatory regions of the RF. This is possible because, in Sillito et al. (1995), the surround grating abutted the center grating, and surround-alone stimulation often activated the cell. In our study, however, there was a gap $\geq 2^{\circ}$ wide between the center and surround gratings. This distance exceeds the anatomical spread of geniculocortical connections (Angelucci and Sainsbury, 2006) and the size of LGN surrounds (Sceniak et al., 2006; Alitto and Usrey, 2008), and, thus, it is unlikely that the surround stimuli directly activated the RFs. Our results on surround facilitation are partly consistent with those of Cavanaugh et al. (2002b) because, unlike Sillito et al. (1995), we found no surround effects when the RF was stimulated with orthogonal-to-optimal orientations. However, unlike both studies, we found that facilitation, although less prevalent than suppression, occurred frequently when the RF and surround were both weakly activated, the RF by stimuli of suboptimal orientation, the surround by small annular gratings. Cavanaugh et al. (2002b) failed to observe facilitation likely because they used fixed-size, large surround gratings for all cells, which we have shown here and previously (Ichida et al., 2007) to evoke predominantly suppression. Instead, we varied the size of the surround grating and analyzed the response of each cell at the specific size that evoked facilitation. Furthermore, the neuronal population of Cavanaugh et al. (2002b) included only broadly orientation-tuned cells. These are unlikely to show facilitatory effects because most oriented stimuli in their RF evoke relatively strong responses, whereas facilitation dominates for weak RF stimulation.

\section{An intracortical mechanism for the orientation dependence of surround modulation in V1: the role of strong local recurrent connections}

What mechanism could adjust the orientation specificity of surround modulation to the stimulus presented to the RF? Cavanaugh et al. (2002a) proposed a simple statistical model of surround suppression, consisting of a center excitatory mechanism overlapping a spatially broader surround inhibitory mechanism, both having Gaussian sensitivity profiles, fixed tuning characteristics, and interacting divisively. These authors argued that the spatial overlap between the center and surround mechanisms implies that any stimulus meant to activate only the RF or the surround, in fact, engages both to some degree. Accordingly, an optimally oriented surround stimulus could increase the response of the center to a suboptimal stimulus, thus exerting relatively less suppression than an iso-oriented suboptimal surround stimulus. However, this is an ad hoc explanation that cannot consistently predict when a stimulus affects the center mechanism more strongly than the surround mechanism or vice versa. Furthermore, this statistical model does not provide a mechanistic explanation of neural responses. Another possibility is that the center and surround mechanisms have specific spatiotemporal phase relationships that result in maximal suppression only when the center and the surround stimuli are in alignment.

Our anatomically realistic recurrent network model of V1 provides a more consistent explanation of these experimental results without assuming any specific spatiotemporal phase relationships between the center and the surround units. This model suggests that strong local recurrent connections, an anatomically prominent feature of the cortex (Binzegger et al., 2004; Stepanyants et al., 2008), play a key role in the computation of surround modulation, by determining the impact of external modulatory inputs. A role for local connections in surround suppression was proposed previously. For example, in the models of Bartsch et al. (2001) and Wielaard and Sajda (2006), surround suppression is mediated by cascades of local connections. However, the latter may contribute to suppression arising from the near, but not from the far, surround because lateral propagation of signals in V1 across many synaptic relays is too slow (Grinvald et al., 1994; Nowak and Bullier, 1997; Bringuier et al., 1999; Girard et al., 2001) to account for the fast onset of far surround suppression (Bair et al., 2003). Theoreticians have long proposed that V1 may operate in a strong recurrent regimen, with balanced excitation and inhibition. Balanced network models have been used to explain the large variability in cortical responses (van Vreeswijk and Sompolinsky, 1996) and can also account for many properties of surround suppression in V1 (Bressloff and Cowan, 2002). These models, however, did not investigate the role of strong recurrent connections in the stimulus-dependent orientation tuning of surround modulation. Recent experimental evidence suggests that V1 may indeed operate like a balanced network (Mariño et al., 2005; Ozeki et al., 2009; Stimberg et al., 2009), possibly an inhibition stabilized network (ISN; Tsodyks et al., 1997; Ozeki et al., 2009) in which strong recurrent excitation would be unstable in the absence of recurrent inhibition. In particular, the ISN model of Ozeki et al. (2009) consists of recurrently connected excitatory (E) and inhibitory (B) neurons, with strong recurrent connections existing only within orientation columns. We have shown that, in this model, strongest suppression occurs when the surround stimulus is at the preferred orientation of the neuron; thus, this model fails to account for the experimentally observed tuning of surround suppression. Instead, in our model, with two populations of inhibitory neurons (I, B) and strong recurrent connections within and between orientation columns, tuned inhibition via the surround pathways causes withdrawal of local recurrent excitation with an accompanying reduction of balanced inhibition. This results in strongest suppression for iso-oriented center and surround stimuli independent of the preferred orientation of the neurons. We argue that highthreshold inhibitory (I) neurons are necessary to account for the contrast dependence of surround modulation (Ichida et al., 2007).

We speculate that this tuning behavior of surround modulation, operating at the population scale, serves to compute visually salient locations in the visual field. It was proposed previously that V1 computes saliency maps ( $\mathrm{Li}, 2002)$, i.e., that all V1 cell responses at a particular visual field location are summed so that outliers are identified as "salient" locations. We predict that, in a $\mathrm{V} 1$ in which surround suppression is strongest at iso-orientation, independent of the RF tuning of the individual cell, salient image locations (e.g., an oriented stimulus embedded in an orthogonally oriented surround) are "more salient" than in a V1 in which surround suppression is dependent on the RF tuning of individual cells. This is because, in the former V1, an iso-oriented centersurround stimulus maximally suppresses all orientation columns across cortex rather than only the columns that are tuned to the specific stimulus orientation, as in the latter V1. As a result, the response difference between a salient image location and less sa- 
lient (iso-oriented regions) locations should be larger in the former than in the latter V1.

\section{References}

Alitto HJ, Usrey WM (2008) Origin and dynamics of extraclassical suppression in the lateral geniculate nucleus of the macaque monkey. Neuron 57:135-146.

Allman J, Miezin F, McGuinness E (1985) Stimulus specific responses from beyond the classical receptive field: neurophysiological mechanisms for local-global comparisons in visual neurons. Annu Rev Neurosci 8:407-430.

Anderson JC, Martin KA (2009) The synaptic connections between cortical areas V1 and V2 in macaque monkey. J Neurosci 29:11283-11293.

Angelucci A, Bressloff PC (2006) The contribution of feedforward, lateral and feedback connections to the classical receptive field center and extraclassical receptive field surround of primate V1 neurons. Prog Brain Res 154:93-120.

Angelucci A, Sainsbury K (2006) Contribution of feedforward thalamic afferents and corticogeniculate feedback to the spatial summation area of macaque V1 and LGN. J Comp Neurol 498:330-351.

Angelucci A, Levitt JB, Walton EJ, Hupe JM, Bullier J, Lund JS (2002) Circuits for local and global signal integration in primary visual cortex. J Neurosci 22:8633-8646.

Bair W, Cavanaugh JR, Movshon JA (2003) Time course and time-distance relationships for surround suppression in macaque V1 neurons. J Neurosci 23:7690-7701.

Bartsch H, Stetter M, Obermayer K (2001) Contextual effects by short range connections in a mean-field model of V1. Neurocomputing $38-40: 475-482$.

Binzegger T, Douglas RJ, Martin KA (2004) A quantitative map of the circuit of cat primary visual cortex. J Neurosci 24:8441-8453.

Blakemore C, Tobin EA (1972) Lateral inhibition between orientation detectors in the cat's visual cortex. Exp Brain Res 15:439-440.

Bressloff PC, Cowan JD (2002) An amplitude equation approach to contextual effects in visual cortex. Neural Comput 14:493-525.

Bringuier V, Chavane F, Glaeser L, Frégnac Y (1999) Horizontal propagation of visual activity in the synaptic integration field of area 17 neurons. Science 283:695-699.

Burkhalter A (2008) Many specialists for suppressing cortical excitation. Front Neurosci 2:155-167.

Cavanaugh JR, Bair W, Movshon JA (2002a) Nature and interaction of signals from the receptive field center and surround in macaque V1 neurons. J Neurophysiol 88:2530-2546.

Cavanaugh JR, Bair W, Movshon JA (2002b) Selectivity and spatial distribution of signals from the receptive field surround in macaque V1 neurons. J Neurophysiol 88:2547-2556.

DeAngelis GC, Freeman RD, Ohzawa I (1994) Length and width tuning of neurons in the cat's primary visual cortex. J Neurophysiol 71:347-374.

Gallant JL, Connor CE, Van Essen DC (1998) Neural activity in areas V1, V2 and V4 during free viewing of natural scenes compared to controlled viewing. Neuroreport 9:2153-2158.

Gilbert CD, Wiesel TN (1990) The influence of contextual stimuli on the orientation selectivity of cells in primary visual cortex of the cat. Vision Res 30:1689-1701.

Girard P, Hupé JM, Bullier J (2001) Feedforward and feedback connections between areas V1 and V2 of the monkey have similar rapid conduction velocities. J Neurophysiol 85:1328-1331.

Gonchar Y, Burkhalter A (2003) Distinct GABAergic targets of feedforward and feedback connections between lower and higher areas of rat visual cortex. J Neurosci 23:10904-10912.

Grinvald A, Lieke EE, Frostig RD, Hildesheim R (1994) Cortical pointspread function and long-range lateral interactions revealed by real-time optical imaging of macaque monkey primary visual cortex. J Neurosci 14:2545-2568.

Ichida JM, Schwabe L, Bressloff PC, Angelucci A (2007) Response facilitation from the "suppressive" receptive field surround of macaque V1 neurons. J Neurophysiol 98:2168-2181.

Kisvárday ZF, Kim DS, Eysel UT, Bonhoeffer T (1994) Relationship between lateral inhibitory connections and the topography of the orientation map in cat visual cortex. Eur J Neurosci 6:1619-1632.

Knierim JJ, van Essen DC (1992) Neuronal responses to static texture patterns in area V1 of the alert macaque monkey. J Neurophysiol 67:961-980.
Lamme VA (1995) The neurophysiology of figure-ground segregation in primary visual cortex. J Neurosci 15:1605-1615.

Levitt JB, Lund JS (1997) Contrast dependence of contextual effects in primate visual cortex. Nature 387:73-76.

Levitt JB, Lund JS (2002) The spatial extent over which neurons in macaque striate cortex pool visual signals. Vis Neurosci 19:439-452.

Li Z (1999) Visual segmentation by contextual influences via intra-cortical interactions in the primary visual cortex. Network 10:187-212.

Li Z (2002) A saliency map in primary visual cortex. Trends Cogn Sci 6:9-16.

Lund JS, Wu CQ (1997) Local circuit neurons of macaque monkey striate cortex. IV. Neurons of laminae 1-3A. J Comp Neurol 384:109-126.

Lund JS, Yoshioka T (1991) Local circuit neurons of macaque monkey striate cortex. III. Neurons of laminae 4B, 4A and 3B. J Comp Neurol 331:234-258.

Lund JS, Wu Q, Hadingham PT, Levitt JB (1995) Cells and circuits contributing to functional properties in area V1 of macaque monkey cerebral cortex: bases for neuroanatomically realistic models. J Anat 187:563-581.

Malach R, Amir Y, Harel M, Grinvald A (1993) Relationship between intrinsic connections and functional architecture revealed by optical imaging and in vivo targeted biocytin injections in primate striate cortex. Proc Natl Acad Sci U S A 90:10469-10473.

Malik J, Perona P (1990) Preattentive texture discrimination with early vision mechanisms. J Opt Soc Am A 7:923-932.

Mariño J, Schummers J, Lyon DC, Schwabe L, Beck O, Wiesing P, Obermayer K, Sur M (2005) Invariant computations in local cortical networks with balanced excitation and inhibition. Nat Neurosci 8:194-201.

McGuire BA, Gilbert CD, Rivlin PK, Wiesel TN (1991) Targets of horizontal connections in macaque primary visual cortex. J Comp Neurol 305:370-392.

Müller JR, Metha AB, Krauskopf J, Lennie P (2003) Local signals from beyond the receptive fields of striate cortical neurons. J Neurophysiol 90:822-831.

Nelson JI, Frost BJ (1978) Orientation selective inhibition from beyond the classical receptive field. Brain Res 139:359-365.

Nowak LG, Bullier J (1997) The timing of information transfer in the visual system. In: Extrastriate cortex in primates (Rockland KS, Kaas JH, Peters A, eds), pp 205-241. New York: Plenum.

Ozeki H, Finn IM, Schaffer ES, Miller KD, Ferster D (2009) Inhibitory stabilization of the cortical network underlies visual surround suppression. Neuron 62:578-592.

Petrov Y, McKee SP (2006) The effect of spatial configuration on surround suppression of contrast sensitivity. J Vis 6:224-238.

Ringach DL, Shapley RM, Hawken MJ (2002) Orientation selectivity in macaque V1: diversity and laminar dependence. J Neurosci 22:5639-5651.

Sceniak MP, Ringach DL, Hawken MJ, Shapley R (1999) Contrast's effect on spatial summation by macaque V1 neurons. Nat Neurosci 2:733-739.

Sceniak MP, Hawken MJ, Shapley R (2001) Visual spatial characterization of macaque V1 neurons. J Neurophysiol 85:1873-1887.

Sceniak MP, Chatterjee S, Callaway EM (2006) Visual spatial summation in macaque geniculocortical afferents. J Neurophysiol 96:3474-3484.

Schwabe L, Obermayer K, Angelucci A, Bressloff PC (2006) The role of feedback in shaping the extra-classical receptive field of cortical neurons: a recurrent network model. J Neurosci 26:9117-9129.

Schwabe L, Ichida JM, Shushruth S, Mangapathy P, Angelucci A (2010) Contrast-dependence of surround suppression in macaque V1: experimental testing of a recurrent network model. Neuroimage 52:777-792.

Sengpiel F, Baddeley RJ, Freeman TC, Harrad R, Blakemore C (1998) Different mechanisms underlie three inhibitory phenomena in cat area 17. Vision Res 38:2067-2080.

Shmuel A, Korman M, Sterkin A, Harel M, Ullman S, Malach R, Grinvald A (2005) Retinotopic axis specificity and selective clustering of feedback projections from V2 to V1 in the owl monkey. J Neurosci 25:2117-2131.

Sillito AM, Grieve KL, Jones HE, Cudeiro J, Davis J (1995) Visual cortical mechanisms detecting focal orientation discontinuities. Nature 378:492-496.

Smith MA, Bair W, Movshon JA (2006) Dynamics of suppression in macaque primary visual cortex. J Neurosci 26:4826-4834.

Solomon SG, White AJ, Martin PR (2002) Extra-classical receptive field 
properties of parvocellular, magnocellular, and koniocellular cells in the primate lateral geniculate nucleus. J Neurosci 22:338-349.

Somers DC, Todorov EV, Siapas AG, Toth LJ, Kim DS, Sur M (1998) A local circuit approach to understanding integration of long-range inputs in primary visual cortex. Cereb Cortex 8:204-217.

Stepanyants A, Hirsch JA, Martinez LM, Kisvárday ZF, Ferecskó AS, Chklovskii DB (2008) Local potential connectivity in cat primary visual cortex. Cereb Cortex 18:13-28.

Stettler DD, Das A, Bennett J, Gilbert CD (2002) Lateral connectivity and contextual interactions in macaque primary visual cortex. Neuron 36:739-750.

Stimberg M, Wimmer K, Martin R, Schwabe L, Mariño J, Schummers J, Lyon DC, Sur M, Obermayer K (2009) The operating regime of local computations in primary visual cortex. Cereb Cortex 19:21662180.

Tsodyks MV, Skaggs WE, Sejnowski TJ, McNaughton BL (1997) Paradoxical effects of external modulation of inhibitory interneurons. J Neurosci 17:4382-4388.

van Vreeswijk C, Sompolinsky H (1996) Chaos in neuronal networks with balanced excitatory and inhibitory activity. Science 274:1724- 1726.

Webb BS, Tinsley CJ, Barraclough NE, Easton A, Parker A, Derrington AM (2002) Feedback from V1 and inhibition from beyond the classical receptive field modulates the responses of neurons in the primate lateral geniculate nucleus. Vis Neurosci 19:583-592.

Wielaard J, Sajda P (2006) Extraclassical receptive field phenomena and short-range connectivity in V1. Cereb Cortex 16:1531-1545. 